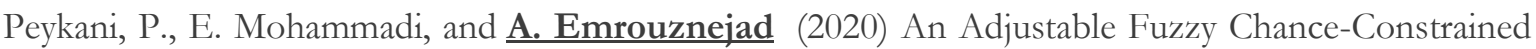
Network DEA Approach with Application to Ranking Investment Firms, Expert Systems with Applications, https://doi.org/10.1016/j.eswa.2020.113938

\title{
An Adjustable Fuzzy Chance-Constrained Network DEA Approach with Application to Ranking Investment Firms
}

\author{
Pejman Peykani' ${ }^{1}$ Emran Mohammadi², Ali Emrouznejad ${ }^{* 3}$
}

${ }^{1}$ School of Industrial Engineering, Iran University of Science and Technology, Tehran, Iran, pejman.peykani@yahoo.com ${ }^{2}$ School of Industrial Engineering, Iran University of Science and Technology, Tehran, Iran, e_mohammadi@iust.ac.ir

${ }^{3}$ Aston Business School, Aston University, Birmingham, UK, a.emrouznejad@aston.ac.uk

\section{Abstract}

This paper presents a novel approach for performance appraisal and ranking of decision-making units (DMUs) with two-stage network structure in the presence of imprecise and vague data. In order to achieve this goal, two-stage data envelopment analysis (DEA) model, adjustable possibilistic programming (APP), and chanceconstrained programming $(\mathrm{CCP})$ are applied to propose the new fuzzy network data envelopment analysis (FNDEA) approach. The main advantages of the proposed FNDEA approach can be summarized as follows: linearity of the proposed FNDEA models, unique efficiency decomposing under ambiguity, capability to extending for other network structures. Moreover, FNDEA approach can be applied for ranking of two-stage DMUs under fuzzy environment in three stages: 1) solving the proposed FNDEA model for all optimistic-pessimistic viewpoints and confidence levels, 2) then plotting the results and drawing the surface of all efficiency scores, 3) and finally calculate the volume of the three-dimensional shape in below the efficiency surface. This volume can be as ranking criterion. Remarkably, the presented fuzzy network DEA approach is implemented for performance appraisal and ranking of investment firms (IFs) with two-stage processes including operational and portfolio management process. Illustrative results of the real-life case study show that the proposed approach is effective and practically very useful.

\footnotetext{
* Corresponding Author: Ali Emrouznejad, Professor of Business Analytics, Aston Business School, Aston University, Birmingham, UK, E-mail: a.emrouznejad@aston.ac.uk, Website: http:/ / www.deazone.com
} 
Keywords: Network Data Envelopment Analysis, Two-Stage Structure, Fuzzy ChanceConstrained Programming, Adjustable Possibilistic Programming, Investment Firms.

\section{Introduction}

Charnes et al. (1978) inspired from Farrell's (1957) propose data envelopment analysis (DEA) technique for measuring the relative efficiency of a set of decision-making units (DMUs) that apply multiple inputs to produce multiple outputs under the assumption of constant returns to scale (Emrouznejad \& Yang, 2018). Their model was later developed by Banker et al. (1984) to measure efficiency under the assumption of variable returns to scale. Traditional DEA models neglect internal or linking activities, but in many cases such as insurance, bank, hotel, airline, manufacturing system, supply chain, etc., DMUs may have internal or network structures, as shown in Figure 1.

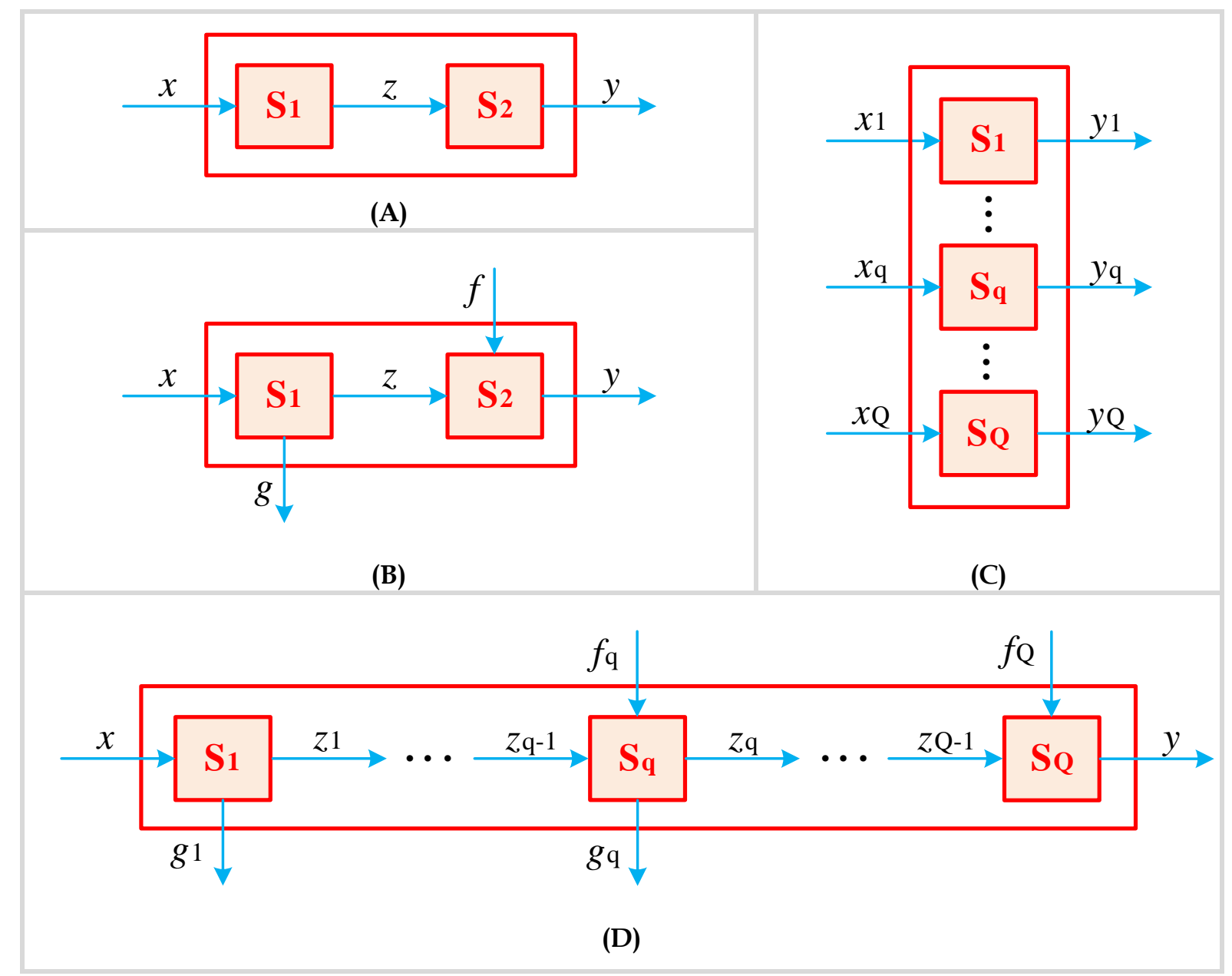

Figure 1. Graphical Prestation of DMU with Network Structures (A) Basic Two-Stage, (B) Extended Two-Stage, (C) Parallel, (D) Series 
Recently, some studies about DEA have focused on DMUs with internal structures which lead to proposing variants of network data envelopment analysis (NDEA) models (Castelli et al., 2010; Cook \& Zhu, 2014). Nowadays, NDEA is a powerful and applicable approach that can be applied for performance assessment of DMUs with network structure such as two-stage, series, parallel, hierarchical, and mixed (Kao, 2014a; 2017).

An important point that should be considered in proposed NDEA models when used in performance assessment of DMUs in real-world problems, is uncertainty, where a little bias or deviation in values cause significant differences in final results (Peykani et al., 2020). Fuzzy network data envelopment analysis (FNDEA) is one of the popular and applicable methods that can be used to efficiency measurement and ranking of DMUs with network structure in the presence of imprecise and vague data.

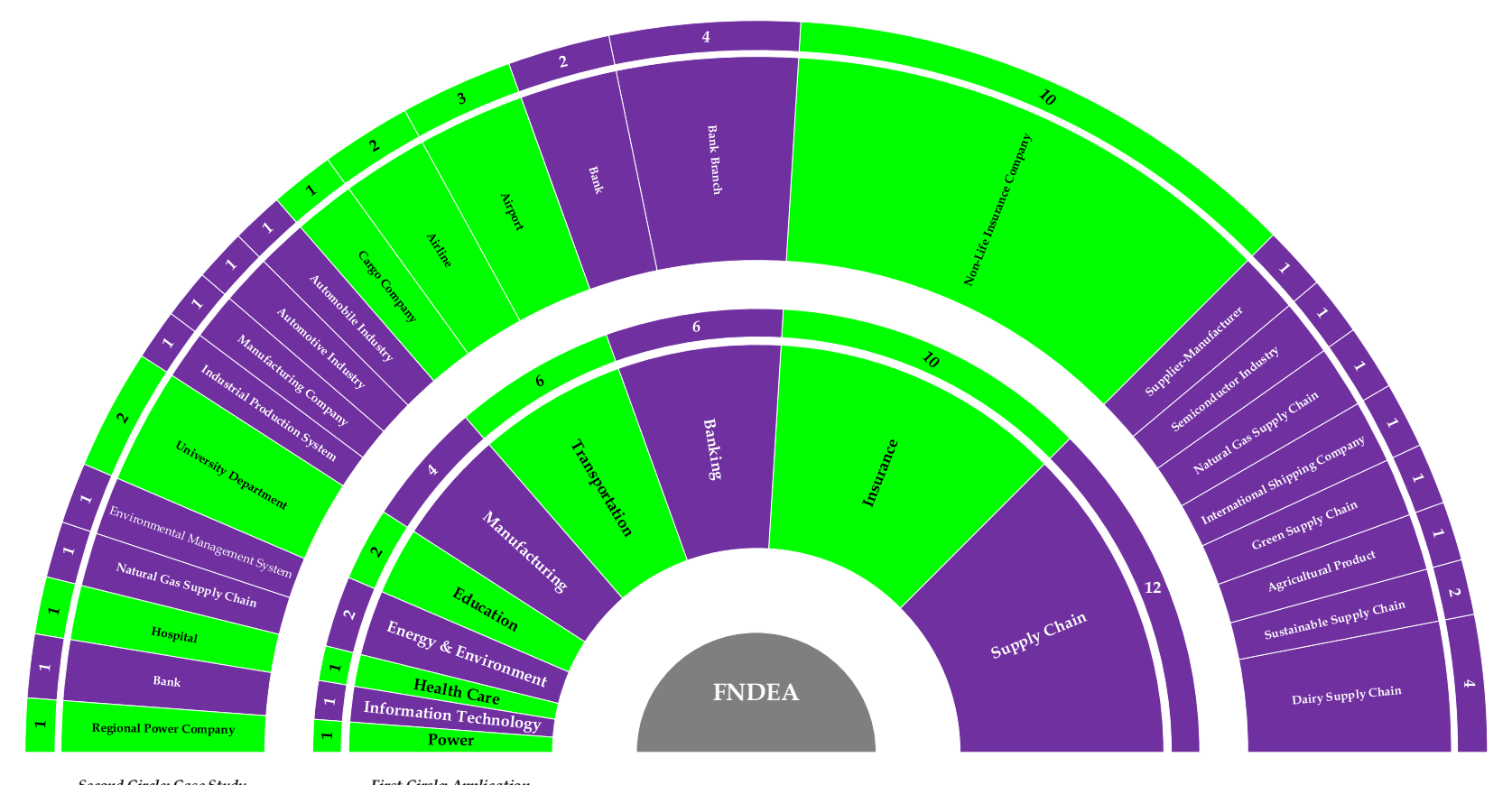

Figure 2. Real-World Applications of FNDEA Approach

As it can be seen in Figure 2, fuzzy network DEA has been widely used in various areas by many researchers and it has become enforceable in different real-world problems. For more details, a literature review and analysis of FNDEA application studies are presented in Table 1. 
Table 1. The Review of FNDEA Studies from Application Viewpoint

\begin{tabular}{|c|c|c|c|c|}
\hline Research & Application & Case Study & Objective* $^{*}$ & $\begin{array}{l}\text { Network } \\
\text { Structure }\end{array}$ \\
\hline Kao \& Liu (2011) & Insurance & $\begin{array}{c}\text { Non-Life Insurance } \\
\text { Company }\end{array}$ & PM & $\begin{array}{c}\text { Basic Two- } \\
\text { Stage }\end{array}$ \\
\hline $\begin{array}{c}\text { Kao \& Lin (2012) } \\
\text { Khalili- }\end{array}$ & Education & University Department & PM & Parallel \\
\hline $\begin{array}{l}\text { Damghani \& } \\
\text { Taghavifard } \\
\text { (2012) }\end{array}$ & Supply Chain & Dairy Supply Chain & PM & Series \\
\hline $\begin{array}{c}\text { Khalili- } \\
\text { Damghani et al. } \\
(2012)\end{array}$ & Supply Chain & Dairy Supply Chain & PM & $\begin{array}{l}\text { Basic Two- } \\
\text { Stage }\end{array}$ \\
\hline $\begin{array}{c}\text { Rostamy- } \\
\text { Malkhalifeh \& } \\
\text { Mollaeian (2012) }\end{array}$ & Supply Chain & Supplier-Manufacturer & PM & Mixed \\
\hline $\begin{array}{c}\text { Khalili- } \\
\text { Damghani \& } \\
\text { Tavana (2013) }\end{array}$ & Supply Chain & Dairy Supply Chain & PM & Mixed \\
\hline $\begin{array}{c}\text { Khalili- } \\
\text { Damghani et al. } \\
(2014)\end{array}$ & Supply Chain & Dairy Supply Chain & PM & $\begin{array}{c}\text { Basic Two- } \\
\text { Stage }\end{array}$ \\
\hline Liu (2014a) & Insurance & $\begin{array}{c}\text { Non-Life Insurance } \\
\text { Company }\end{array}$ & Ranking & $\begin{array}{l}\text { Basic Two- } \\
\text { Stage }\end{array}$ \\
\hline Liu (2014b) & $\begin{array}{l}\text { Insurance / } \\
\text { Banking \& } \\
\text { Information } \\
\text { Technology }\end{array}$ & $\begin{array}{c}\text { Non-Life Insurance } \\
\text { Company / Bank }\end{array}$ & PM & $\begin{array}{c}\text { Basic Two- } \\
\text { Stage }\end{array}$ \\
\hline Lozano (2014) & Insurance & $\begin{array}{c}\text { Non-Life Insurance } \\
\text { Company }\end{array}$ & PM & $\begin{array}{c}\text { Basic Two- } \\
\text { Stage }\end{array}$ \\
\hline $\begin{array}{c}\text { Lozano \& } \\
\text { Moreno (2014) }\end{array}$ & Insurance & $\begin{array}{c}\text { Non-Life Insurance } \\
\text { Company }\end{array}$ & PM & $\begin{array}{c}\text { Basic Two- } \\
\text { Stage }\end{array}$ \\
\hline $\begin{array}{l}\text { Mirhedayatian et } \\
\text { al. (2014) }\end{array}$ & Supply Chain & Green Supply Chain & PM & Series \\
\hline $\begin{array}{l}\text { Momeni et al. } \\
\text { (2014) }\end{array}$ & Supply Chain & Semiconductor Industry & PM & Series \\
\hline $\begin{array}{c}\text { Tavana \& } \\
\text { Khalili- } \\
\text { Damghani (2014) }\end{array}$ & Banking & Bank Branch & PM & $\begin{array}{c}\text { Basic Two- } \\
\text { Stage }\end{array}$ \\
\hline Xia et al. (2014) & Banking & Bank Branch & PM & $\begin{array}{l}\text { Basic Two- } \\
\text { Stage }\end{array}$ \\
\hline $\begin{array}{l}\text { Hemmati et al. } \\
\text { (2016) }\end{array}$ & Manufacturing & $\begin{array}{c}\text { Manufacturing } \\
\text { Company }\end{array}$ & PM & $\begin{array}{c}\text { Basic Two- } \\
\text { Stage }\end{array}$ \\
\hline Olfat et al. (2016) & Transportation & Airport & PM & Mixed \\
\hline $\begin{array}{l}\text { Shermeh et al. } \\
\text { (2016) }\end{array}$ & Power & $\begin{array}{l}\text { Regional Power } \\
\text { Company }\end{array}$ & PM & Series \\
\hline $\begin{array}{l}\text { Olfat \& Pishdar } \\
\text { (2017) }\end{array}$ & Transportation & Airport & $\mathrm{PM}$ & $\begin{array}{l}\text { Basic Two- } \\
\text { Stage }\end{array}$ \\
\hline $\begin{array}{l}\text { Yousefi et al. } \\
\text { (2017) }\end{array}$ & Supply Chain & $\begin{array}{c}\text { Sustainable Supply } \\
\text { Chain }\end{array}$ & PM & Mixed \\
\hline $\begin{array}{l}\text { Hatami-Marbini } \\
\text { \& Saati (2018) }\end{array}$ & Insurance & $\begin{array}{l}\text { Non-Life Insurance } \\
\text { Company }\end{array}$ & PM & $\begin{array}{l}\text { Basic Two- } \\
\text { Stage }\end{array}$ \\
\hline $\begin{array}{l}\text { Hatami-Marbini } \\
\text { et al. (2018) }\end{array}$ & Insurance & $\begin{array}{c}\text { Non-Life Insurance } \\
\text { Company }\end{array}$ & PM & $\begin{array}{c}\text { Basic Two- } \\
\text { Stage }\end{array}$ \\
\hline $\begin{array}{l}\text { Omrani et al. } \\
\text { (2018) }\end{array}$ & Supply Chain & $\begin{array}{c}\text { International Shipping } \\
\text { Company }\end{array}$ & PM & Series \\
\hline $\begin{array}{l}\text { Şimşek \& Tüysüz } \\
\text { (2018) }\end{array}$ & Transportation & Cargo Company & PM & $\begin{array}{c}\text { Basic Two- } \\
\text { Stage }\end{array}$ \\
\hline
\end{tabular}




\begin{tabular}{|c|c|c|c|c|}
\hline Research & Application & Case Study & Objective* $^{*}$ & $\begin{array}{l}\text { Network } \\
\text { Structure }\end{array}$ \\
\hline $\begin{array}{l}\text { Soltanzadeh \& } \\
\text { Omrani (2018) }\end{array}$ & Transportation & Airline & PM & $\begin{array}{l}\text { Extended Two- } \\
\text { Stage }\end{array}$ \\
\hline $\begin{array}{l}\text { Tavana et al. } \\
\qquad(2018)\end{array}$ & Banking & Bank Branch & PM & $\begin{array}{l}\text { Basic Two- } \\
\text { Stage }\end{array}$ \\
\hline Zhou et al. (2018) & $\begin{array}{c}\text { Manufacturing \& } \\
\text { Environmental }\end{array}$ & $\begin{array}{c}\text { Industrial Production \& } \\
\text { Environmental } \\
\text { Management System }\end{array}$ & PM / Ranking & $\begin{array}{l}\text { Extended Two- } \\
\text { Stage }\end{array}$ \\
\hline $\begin{array}{l}\text { Ameri et al. } \\
\text { (2019) }\end{array}$ & Health Care & Hospital & PM & Parallel \\
\hline $\begin{array}{l}\text { Hatami-Marbini } \\
\qquad(2019)\end{array}$ & Transportation & Airport & PM & $\begin{array}{l}\text { Basic Two- } \\
\text { Stage }\end{array}$ \\
\hline Li et al. (2019) & Manufacturing & Automotive Industry & PM & Mixed \\
\hline $\begin{array}{c}\text { Nasseri \& Khatir } \\
\text { (2019) }\end{array}$ & Banking & Bank Branch & PM & $\begin{array}{l}\text { Basic Two- } \\
\text { Stage }\end{array}$ \\
\hline $\begin{array}{l}\text { Nosrat et al. } \\
\text { (2019) }\end{array}$ & Insurance & $\begin{array}{l}\text { Non-Life Insurance } \\
\text { Company }\end{array}$ & PM & $\begin{array}{l}\text { Basic Two- } \\
\text { Stage }\end{array}$ \\
\hline $\begin{array}{l}\text { Sarah \& Khalili- } \\
\text { Damghani (2019) }\end{array}$ & $\begin{array}{l}\text { Supply Chain \& } \\
\text { Energy }\end{array}$ & $\begin{array}{c}\text { Natural Gas Supply } \\
\text { Chain }\end{array}$ & PM & Mixed \\
\hline $\begin{array}{l}\text { Singh \& Arya } \\
\text { (2019) }\end{array}$ & Insurance & $\begin{array}{l}\text { Non-Life Insurance } \\
\text { Company }\end{array}$ & PM & Mixed \\
\hline $\begin{array}{l}\text { Tabasi et al. } \\
\text { (2019) }\end{array}$ & Manufacturing & Automobile Industry & PM & $\begin{array}{l}\text { Basic Two- } \\
\text { Stage }\end{array}$ \\
\hline $\begin{array}{l}\text { Zhou et al. } \\
\text { (2019a) }\end{array}$ & Supply Chain & $\begin{array}{l}\text { Sustainable Supply } \\
\text { Chain }\end{array}$ & PM & Series \\
\hline $\begin{array}{l}\text { Zhou et al. } \\
\text { (2019b) }\end{array}$ & Banking & Bank & PM & Series \\
\hline $\begin{array}{l}\text { Amirteimoori et } \\
\text { al. (2020) }\end{array}$ & Insurance & $\begin{array}{c}\text { Non-Life Insurance } \\
\text { Company }\end{array}$ & PM / Ranking & $\begin{array}{l}\text { Basic Two- } \\
\text { Stage }\end{array}$ \\
\hline $\begin{array}{l}\text { Ostovan et al. } \\
\qquad(2020)\end{array}$ & Transportation & Airline & PM & $\begin{array}{l}\text { Basic Two- } \\
\text { Stage }\end{array}$ \\
\hline Shi et al. (2020) & Education & University Department & PM & Parallel \\
\hline $\begin{array}{l}\text { Wang and Yao } \\
(2020)\end{array}$ & Supply Chain & Agricultural Product & PM & Series \\
\hline Our Work & Finance & Mutual Fund & PM / Ranking & $\begin{array}{l}\text { Extended Two- } \\
\text { Stage }\end{array}$ \\
\hline
\end{tabular}

${ }^{*} \mathrm{PM}=$ Performance Measurement

In the following, a briefly review of prominent FNDEA studies are introduced. Lotfi et al. (2009) extended a multi-activity network data envelopment analysis in the presence of triangular fuzzy inputs and outputs and also applied a ranking function to propose equivalent crisp of multi-activity network fuzzy DEA. Kao \& Liu (2011) applied $a$-cut approach for two-stage DEA in the presence of fuzzy data and implement their model in non-life insurance companies of Taiwan. Khalili-Damghani \& Taghavifard (2012) proposed a three-stage fuzzy data envelopment analysis for measuring the performance of a serial process including just-in-time (JIT) practices, agility indices and goals in dairy supply chains.

Khalili-Damghani et al. (2012) introduced a fuzzy network DEA with two-stage structure for agility performance measurement in dairy supply chains under 
uncertainty. Khalili-Damghani \& Tavana (2013) presented a fuzzy network DEA model for efficiency measurement of agility in supply chains. Lozano \& Moreno (2013) extended a DEA model for two-stage process in the presence of fuzzy data. Kao (2014b) applied the membership grade and the a-cut methods for measuring the efficiency of network DEA approach in the presence of fuzzy data. Liu (2014a) presented a method to rank the network DMUs with two-stage structure in the presence of fuzzy data. Liu (2014b) introduced a fuzzy two-stage DEA approach under weight restrictions and fuzzy environment. Lozano \& Moreno (2014) generalized several fuzzy DEA approaches to the network DEA context in order to handle fuzzy data when the decision-making units are formed by a network structure.

Mirhedayatian et al. (2014) presented a new network data envelopment analysis model for assessing the firms in green supply chain management (GSCM), which encompasses dual-role factors, undesirable outputs, and fuzzy data. Momeni et al. (2014) introduced a novel fuzzy network slacks-based measure (FNSBM) for performance evaluation of supply chain networks with forward and reverse logistics. Tavana \& Khalili-Damghani (2014) introduced a two-stage fuzzy network DEA model using Stackelberg (non-cooperative or leader-follower) game theory approach and implemented their model in the banking industry.

Wang et al. (2014) used fuzzy multi-objective programming (FMOP) approaches for proposing a fuzzy multi-objective two-stage DEA in order to performance assessment of bank holding companies (BHCs) in United State of America. Xia et al. (2014) integrated fuzzy intermediate factors with triangular fuzzy membership function in supply chain performance measurement and proposed a fuzzy supply chain DEA. Shermeh et al. (2016) presented a fuzzy network slacks-based measure model for efficiency measurement of regional power companies in Iran under fuzzy data.

Olfat et al. (2016) proposed a fuzzy dynamic network data envelopment analysis (FDNDEA) approach for airports performance measurement. Soltanzadeh \& Omrani (2018) extended the dynamic network DEA model in a fuzzy framework and use a case study of airlines in Iran to illustrate the capability of the proposed fuzzy dynamic NDEA model. Tavana et al. (2018) introduced a fuzzy two-stage DEA model using a 
bargaining game approach and used the proposed model for performance assessment of Saman bank branches in Iran by considering their productivity and profitability processes. Ameri et al. (2019) introduced self-assessment method to calculate the efficiency of parallel network systems such as hospitals under intuitionistic fuzzy situations.

Hatami-Marbini (2019) presented a new FNDEA model based on the fuzzy arithmetic approach and applied this model for benchmarking of airport and travel sector. Li et al. (2019) proposed a fuzzy network epsilon-based DEA approach for supply chain performance assessment. Sarah \& Khalili-Damghani (2019) applied fuzzy type-2 De-Novo programming for resource allocation and target setting in NDEA in the presence of uncertainty. Amirteimoori et al. (2020) proposed double frontier fuzzy network DEA approach for performance assessment two-stage DMUs. More recently, Shi et al. (2020) presented a FNDEA model for measuring the efficiency of parallel systems using Stackelberg game theory.

After survey the FNDEA studies and by inspiring of Hatami-Marbini, et al. (2011) and Emrouznejad \& Tavana (2014), the fuzzy network DEA modeling approaches can be classified into six categories that presented in Figure 3.

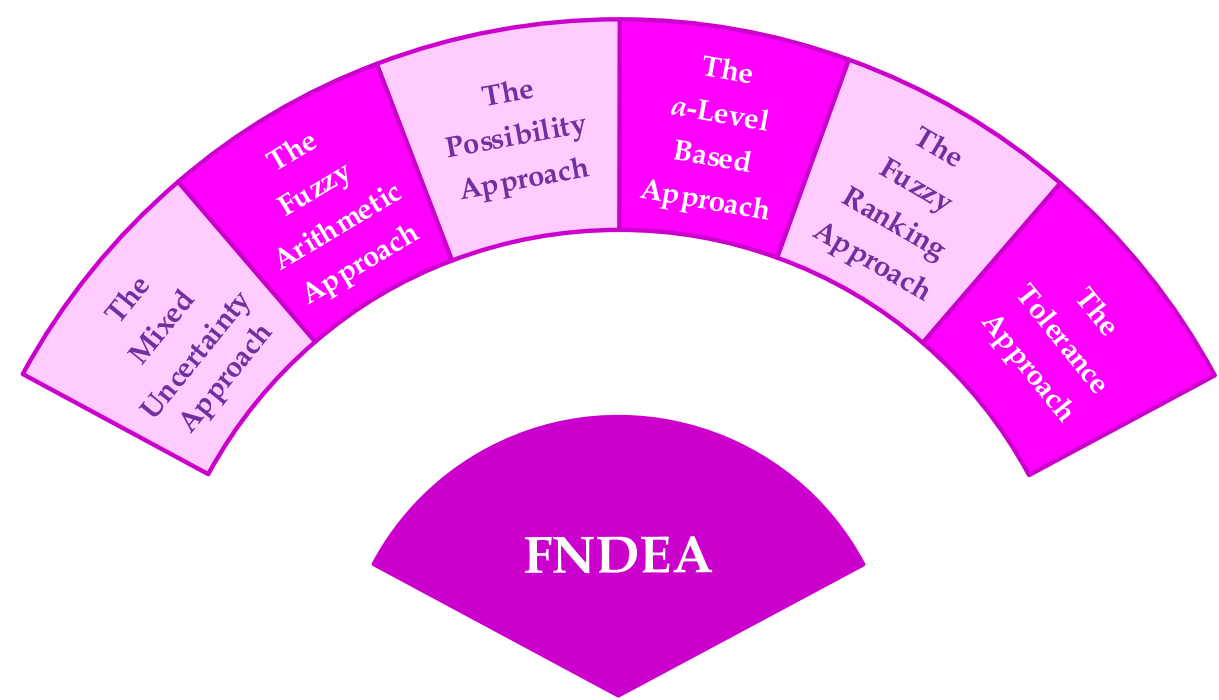

Figure 3. The Classification of FNDEA Approach

It is important to note that most of the FNDEA models are presented based on multiple efficiency decomposition approach, while this approach is not capable to be 
used for complex network structures such as general two-stage structure with additional inputs and/or leakage variables.

Accordingly, for eliminating the mentioned issues, in this study a novel fuzzy network DEA model is proposed based on additive efficiency decomposition approach by applying adjustable possibilistic programming (APP) and chanceconstrained programming (CCP). Additionally, the proposed adjustable fuzzy chance-constrained network DEA model is employed to present a new ranking method for network DMUs with two-stage structure in the presence of fuzzy data. Notably, the proposed FNDEA approach is modeled in a linear form and it is capable to be extended for series and parallel structures.

Finally, the proposed fuzzy network DEA approach is implemented for a real case study of Iranian financial market in order to performance evaluation of investment firms (IFs). It should be noted that investment firms such as mutual funds (MFs) are very important institutions for investing in capital markets. Hence, evaluating their performance with the aim of identifying efficient investment firms and providing a corrective remedy for inefficient IFs is essential.

The rest of this paper is organized as follows. The modelling of basic NDEA model based on additive efficiency decomposition for general two-stage structure will be explained in Section 2. The novel FNDEA model based on adjustable possibilistic programming will be proposed in Section 3. The new approach for ranking of twostage DMUs under fuzzy data is presented in Section 4 . Then, the proposed approach and models in this study are implemented for a real case study of mutual funds and the results will be evaluated in Section 5. Finally, the discussion and conclusions of study are given in Section 6 .

\section{Two-Stage Data Envelopment Analysis Model}

The two-stage structure is one of the popular network structures that has been widely discussed in the NDEA literature (Cook et al., 2010a; Halkos et al., 2014). Figure 4 graphically illustrates an general two-stage structure, where there are a set of $n$ homogenous decision making units $\operatorname{DMU}_{j}(j=1, \ldots, n)$ where each DMU has $I$ inputs 
$x_{i j}(i=1, \ldots, I)$ in the first stage, $K$ outputs (leakage variable) $f_{k j}(k=1, \ldots, K)$ that leave the system in the first stage, $D$ intermediate variables $z_{d j}(d=1, \ldots, D)$ that link first and second stage, $H$ additional inputs $g_{h j}(h=1, \ldots, H)$ in the second stage and finally $R$ outputs $y_{r j}(r=1, \ldots, R)$ in the second stage.

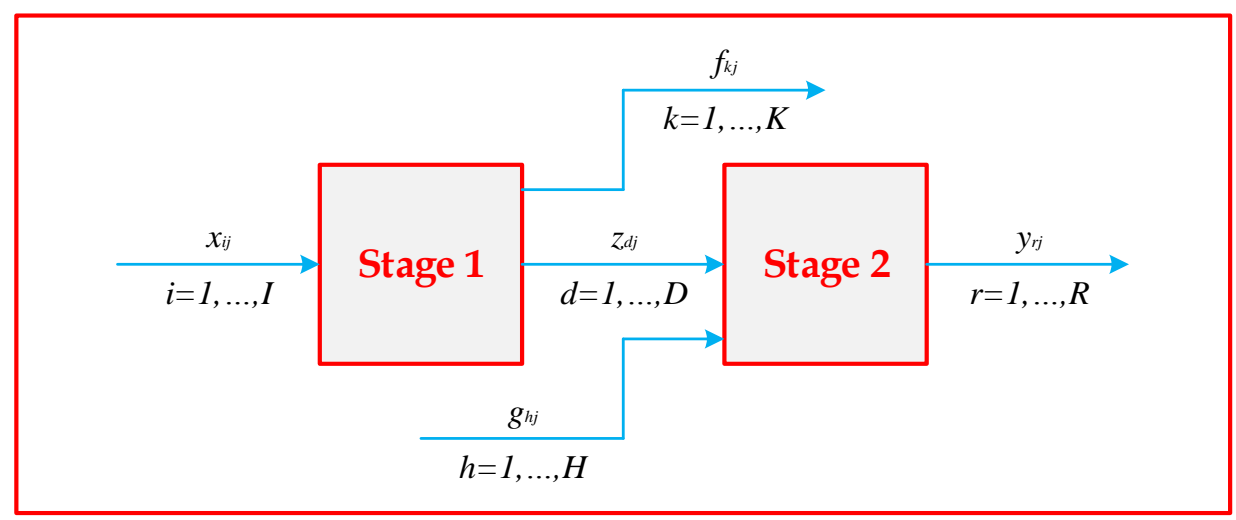

Figure 4. General Two-Stage Structure

Also, the non-negative weights $\tau_{i}(i=1, \ldots, I), \omega_{k}(k=1, \ldots, K), \quad \gamma_{d}(d=1, \ldots, D)$, $\pi_{h}(h=1, \ldots, H)$, and $\mu_{r}(r=1, \ldots, R)$ are assigned to the $x_{i j}(i=1, \ldots, I), f_{k j}(k=1, \ldots, K)$, $z_{d j}(d=1, \ldots, D), g_{h j}(h=1, \ldots, H)$, and $y_{r j}(r=1, \ldots, R)$, respectively.

It should be mentioned that that in NDEA literature, different methods such as game-theoretic (cooperative and non-cooperative game) approach (Liang et al., 2008), multiple efficiency decomposition approach (Kao \& Hwang, 2008), and additive efficiency decomposition approach (Chen et al., 2009) are proposed for NDEA modeling. In this study, the additive efficiency decomposition approach is applied as a basic NDEA method to performance measurement of DMUs (system) and subDMUs (stages 1 and 2). Because, this approach is capable to be used for a general twostage structure with leakage variable in the first stage and added inputs to the second stage. Notably, the additive efficiency decomposition approach is one of the popular methods in network DEA area (Kao, 2014a; 2017).

In the following, the modeling procedure based on additive approach for general two-stage structure is described. According to the Figure 4, the efficiency score of first 
stage and second stage for DMU under investigation $\left(\mathrm{DMU}_{p}\right)$, can be calculated by the following Models (1) and (2), respectively:

$\Psi_{p}^{\text {Stage } 1}=\operatorname{Max} \frac{\sum_{k=1}^{K} f_{k p} \omega_{k}+\sum_{d=1}^{D} z_{d p} \gamma_{d}}{\sum_{i=1}^{I} x_{i p} \tau_{i}}$

S.t. $\quad \frac{\sum_{k=1}^{K} f_{k j} \omega_{k}+\sum_{d=1}^{D} z_{d j} \gamma_{d}}{\sum_{i=1}^{I} x_{i j} \tau_{i}} \leq 1, \quad \forall j$

$\tau_{i}, \omega_{k}, \gamma_{d} \geq 0, \quad \forall i, k, d$

$\Psi_{p}^{\text {Stage2 }}=\operatorname{Max} \frac{\sum_{r=1}^{R} y_{r p} \mu_{r}}{\sum_{d=1}^{D} z_{d p} \gamma_{d}+\sum_{h=1}^{H} g_{h p} \pi_{h}}$

S.t. $\quad \frac{\sum_{r=1}^{R} y_{r j} \mu_{r}}{\sum_{d=1}^{D} z_{d j} \gamma_{d}+\sum_{h=1}^{H} g_{h j} \pi_{h}} \leq 1, \quad \forall j$

$\gamma_{d}, \pi_{h}, \mu_{r} \geq 0, \quad \forall d, h, r$

According to the idea of Chen et al. (2009), the overall efficiency of the general two-stage process can be defined as Equation (3):

$$
\Psi_{p}^{\text {Overall }}=\xi_{1}\left(\Psi_{p}^{\text {Stage1 }}\right)+\xi_{2}\left(\Psi_{p}^{\text {Stage2 }}\right)=\xi_{1}\left(\frac{\sum_{k=1}^{K} f_{k p} \omega_{k}+\sum_{d=1}^{D} z_{d p} \gamma_{d}}{\sum_{i=1}^{I} x_{i p} \tau_{i}}\right)+\xi_{2}\left(\frac{\sum_{r=1}^{R} y_{r p} \mu_{r}}{\sum_{d=1}^{D} z_{d p} \gamma_{d}+\sum_{h=1}^{H} g_{h p} \pi_{h}}\right)
$$

Note that in Equation (3), $\xi_{1}$ and $\xi_{2}$ are user-specified weights such that $\xi_{1}+\xi_{2}=1$. In other words, $\xi_{1}$ and $\xi_{2}$ indicate the relative importance of the performances of stages 1 and 2, respectively, to the overall performance of the system. 
Accordingly, the overall efficiency score of $\mathrm{DMU}_{p}$ is estimated by solving the Model (4) as follows:

$\Psi_{p}^{\text {Overall }}=\operatorname{Max} \xi_{1}\left(\frac{\sum_{k=1}^{K} f_{k p} \omega_{k}+\sum_{d=1}^{D} z_{d p} \gamma_{d}}{\sum_{i=1}^{I} x_{i p} \tau_{i}}\right)+\xi_{2}\left(\frac{\sum_{r=1}^{R} y_{r p} \mu_{r}}{\sum_{d=1}^{D} z_{d p} \gamma_{d}+\sum_{h=1}^{H} g_{h p} \pi_{h}}\right)$

S.t. $\quad \frac{\sum_{k=1}^{K} f_{k j} \omega_{k}+\sum_{d=1}^{D} z_{d j} \gamma_{d}}{\sum_{i=1}^{I} x_{i j} \tau_{i}} \leq 1, \quad \forall j$

$$
\begin{aligned}
& \frac{\sum_{r=1}^{R} y_{r j} \mu_{r}}{\sum_{d=1}^{D} z_{d j} \gamma_{d}+\sum_{h=1}^{H} g_{h j} \pi_{h}} \leq 1, \quad \forall j \\
& \tau_{i}, \omega_{k}, \gamma_{d}, \pi_{h}, \mu_{r} \geq 0, \quad \forall i, k, d, h, r
\end{aligned}
$$

As it can be seen in Model (4), this model cannot be converted into a linear program (LP) by applying the usual Charnes and Cooper (1962) transformation. For eliminating this issue, Chen et al. (2009) suggested $\xi_{1}$ and $\xi_{2}$ are defined as Equations (5) and (6), respectively:

$$
\begin{aligned}
& \xi_{1}=\frac{\sum_{i=1}^{I} x_{i p} \tau_{i}}{\sum_{i=1}^{I} x_{i p} \tau_{i}+\sum_{k=1}^{K} f_{k p} \omega_{k}+\sum_{d=1}^{D} z_{d p} \gamma_{d}} \\
& \xi_{2}=\frac{\sum_{k=1}^{K} f_{k p} \omega_{k}+\sum_{d=1}^{D} z_{d p} \gamma_{d}}{\sum_{i=1}^{I} x_{i p} \tau_{i}+\sum_{k=1}^{K} f_{k p} \omega_{k}+\sum_{d=1}^{D} z_{d p} \gamma_{d}}
\end{aligned}
$$

Thus, by applying the above equations, Model (4) will be converted to Model (7) as follows:

$$
\Psi_{p}^{\text {Overall }}=\operatorname{Max} \frac{\sum_{k=1}^{K} f_{k p} \omega_{k}+\sum_{d=1}^{D} z_{d p} \gamma_{d}+\sum_{r=1}^{R} y_{r p} \mu_{r}}{\sum_{i=1}^{I} x_{i p} \tau_{i}+\sum_{d=1}^{D} z_{d p} \gamma_{d}+\sum_{h=1}^{H} g_{h p} \pi_{h}}
$$




$$
\begin{array}{ll}
\text { S.t. } \quad & \frac{\sum_{k=1}^{K} f_{k j} \omega_{k}+\sum_{d=1}^{D} z_{d j} \gamma_{d}}{\sum_{i=1}^{I} x_{i j} \tau_{i}} \leq 1, \quad \forall j \\
& \frac{\sum_{r=1}^{R} y_{r j} \mu_{r}}{\sum_{d=1}^{D} z_{d j} \gamma_{d}+\sum_{h=1}^{H} g_{h j} \pi_{h}} \leq 1, \quad \forall j \\
& \tau_{i}, \omega_{k}, \gamma_{d}, \pi_{h}, \mu_{r} \geq 0, \quad \forall i, k, d, h, r
\end{array}
$$

Now, by employing Charnes and Cooper (1962) transformation, Model (7) can be turned into a LP as Model (8):

$$
\begin{aligned}
& \Psi_{p}^{\text {Overall }}=\operatorname{Max} \sum_{k=1}^{K} f_{k p} \omega_{k}+\sum_{d=1}^{D} z_{d p} \gamma_{d}+\sum_{r=1}^{R} y_{r p} \mu_{r} \\
& \text { S.t. } \quad \sum_{i=1}^{I} x_{i p} \tau_{i}+\sum_{d=1}^{D} z_{d p} \gamma_{d}+\sum_{h=1}^{H} g_{h p} \pi_{h}=1 \\
& \sum_{k=1}^{K} f_{k j} \omega_{k}+\sum_{d=1}^{D} z_{d j} \gamma_{d}-\sum_{i=1}^{I} x_{i j} \tau_{i} \leq 0, \quad \forall j \\
& \sum_{r=1}^{R} y_{r j} \mu_{r}-\sum_{d=1}^{D} z_{d j} \gamma_{d}-\sum_{h=1}^{H} g_{h j} \pi_{h} \leq 0, \quad \forall j \\
& \tau_{i}, \omega_{k}, \gamma_{d}, \pi_{h}, \mu_{r} \geq 0, \quad \forall i, k, d, h, r
\end{aligned}
$$

Note that the optimal multipliers that are solved from Model (8) may not be unique and consequently, the decomposition of the overall efficiency defined in Equation (3) would not be unique. Kao and Hwang (2008) suggested a method to find a set of multipliers which produces the maximum efficiency score for stage 1 (or stage 2) while maintaining the overall efficiency score. If assumed that the efficiency of the first stage is more important for the DM, $\Psi_{p}^{\text {Stage } 1}$ will be estimated by solving Model (9) while $\Psi_{p}^{\text {Overall* }}$ is optimized from Model (8).

$$
\Psi_{p}^{\text {Stagel }}=\operatorname{Max} \frac{\sum_{k=1}^{K} f_{k p} \omega_{k}+\sum_{d=1}^{D} z_{d p} \gamma_{d}}{\sum_{i=1}^{I} x_{i p} \tau_{i}}
$$




$$
\begin{array}{ll}
\text { S.t. } \quad & \frac{\sum_{k=1}^{K} f_{k j} \omega_{k}+\sum_{d=1}^{D} z_{d j} \gamma_{d}}{\sum_{i=1}^{I} x_{i j} \tau_{i}} \leq 1, \quad \forall j \\
& \frac{\sum_{r=1}^{R} y_{r j} \mu_{r}}{\sum_{d=1}^{D} z_{d j} \gamma_{d}+\sum_{h=1}^{H} g_{h j} \pi_{h}} \leq 1, \quad \forall j \\
& \frac{\sum_{k=1}^{K} f_{k p} \omega_{k}+\sum_{d=1}^{D} z_{d p} \gamma_{d}+\sum_{r=1}^{R} y_{r p} \mu_{r}}{\sum_{i=1}^{I} x_{i p} \tau_{i}+\sum_{d=1}^{D} z_{d p} \gamma_{d}+\sum_{h=1}^{H} g_{h p} \pi_{h}}=\Psi_{p}^{\text {Overall* }} \\
& \tau_{i}, \omega_{k}, \gamma_{d}, \pi_{h}, \mu_{r} \geq 0, \quad \forall i, k, d, h, r
\end{array}
$$

Since Model (9) is a linear fractional program (LFP), utilizing the transformation of Charnes and Cooper (1962), this model will be equivalent to Model (10):

$$
\Psi_{p}^{\text {Stage } 1}=\operatorname{Max} \sum_{k=1}^{K} f_{k p} \omega_{k}+\sum_{d=1}^{D} z_{d p} \gamma_{d}
$$

S.t. $\quad \sum_{i=1}^{I} x_{i p} \tau_{i}=1$

$$
\begin{aligned}
& \sum_{k=1}^{K} f_{k j} \omega_{k}+\sum_{d=1}^{D} z_{d j} \gamma_{d}-\sum_{i=1}^{I} x_{i j} \tau_{i} \leq 0, \quad \forall j \\
& \sum_{r=1}^{R} y_{r j} \mu_{r}-\sum_{d=1}^{D} z_{d j} \gamma_{d}-\sum_{h=1}^{H} g_{h j} \pi_{h} \leq 0, \quad \forall j \\
& \sum_{k=1}^{K} f_{k p} \omega_{k}+\sum_{d=1}^{D} z_{d p} \gamma_{d}+\sum_{r=1}^{R} y_{r p} \mu_{r}-\Psi_{p}^{\text {Overall }^{*}} \sum_{d=1}^{D} z_{d p} \gamma_{d}-\Psi_{p}^{\text {Overall }} \sum_{h=1}^{H} g_{h p} \pi_{h}=\Psi_{p}^{\text {Overall } *} \\
& \tau_{i}, \omega_{k}, \gamma_{d}, \pi_{h}, \mu_{r} \geq 0, \quad \forall i, k, d, h, r
\end{aligned}
$$

After calculating $\Psi_{p}^{\text {Stage }{ }^{*}}$ using the Model (10), the efficiency score of the second stage is obtained from Equation (11):

$$
\Psi_{p}^{{\text {Stage } 2^{*}}^{*}}=\frac{\Psi_{p}^{\text {Overall }}-\xi_{1}^{*} \Psi_{p}^{\text {Stage }{ }^{*}}}{\xi_{2}^{*}}
$$

It is worth noting that $\xi_{1}^{*}$ and $\xi_{2}^{*}$ are optimal weights that obtained from Model (8) using Equations (5) and (6). Alternatively, if assumed that the second stage is more 
important, the efficiency of stages 2 and 1 will be calculated in a similar manner that is presented in Appendix A.

\section{A Novel Fuzzy Network Data Envelopment Analysis Model}

Now, in this section, the new fuzzy network DEA model for dealing with imprecise and vague data will be proposed. Notably, for presenting FNDEA model based on additive approach, it assumed that the stage 1 is more important. Accordingly, suppose that all data are approximately known, and are represented by fuzzy numbers $\tilde{x}_{i j}, \tilde{f}_{k j}, \quad \tilde{z}_{d j}, \quad \tilde{g}_{h j}$ and $\tilde{y}_{r j}$ with trapezoidal membership functions $\tilde{x}_{i j}\left(x_{i j}^{1}, x_{i j}^{2}, x_{i j}^{3}, x_{i j}^{4}\right), \quad \tilde{f}_{k j}\left(f_{k j}^{1}, f_{k j}^{2}, f_{k j}^{3}, f_{k j}^{4}\right), \quad \tilde{z}_{d j}\left(z_{d j}^{1}, z_{d j}^{2}, z_{d j}^{3}, z_{d j}^{4}\right), \quad \tilde{g}_{h j}\left(g_{h j}^{1}, g_{h j}^{2}, g_{h j}^{3}, g_{h j}^{4}\right), \quad$ and $\tilde{y}_{r j}\left(y_{r j}^{1}, y_{r j}^{2}, y_{r j}^{3}, y_{r j}^{4}\right)$ in which $x_{i j}^{1}<x_{i j}^{2}<x_{i j}^{3}<x_{i j}^{4}, \quad f_{k j}^{1}<f_{k j}^{2}<f_{k j}^{3}<f_{k j}^{4}, \quad z_{d j}^{1}<z_{d j}^{2}<z_{d j}^{3}<z_{d j}^{4}$, $g_{h j}^{1}<g_{h j}^{2}<g_{h j}^{3}<g_{h j}^{4}$, and $y_{r j}^{1}<y_{r j}^{2}<y_{r j}^{3}<y_{r j}^{4}$. To consider the uncertainty on all data, Models (8) and (10) for fuzzy observations can be formulated as Models (12) and (13), respectively:

$\tilde{\Psi}_{p}^{\text {Overall }}=\operatorname{Max} \Omega$

S.t. $\quad \sum_{k=1}^{K} \tilde{f}_{k p} \omega_{k}+\sum_{d=1}^{D} \tilde{z}_{d p} \gamma_{d}+\sum_{r=1}^{R} \tilde{y}_{r p} \mu_{r} \geq \Omega$

$\sum_{i=1}^{I} \tilde{x}_{i p} \tau_{i}+\sum_{d=1}^{D} \tilde{z}_{d p} \gamma_{d}+\sum_{h=1}^{H} \tilde{g}_{h p} \pi_{h} \leq 1$

$\sum_{k=1}^{K} \tilde{f}_{k j} \omega_{k}+\sum_{d=1}^{D} \tilde{z}_{d j} \gamma_{d}-\sum_{i=1}^{I} \tilde{x}_{i j} \tau_{i} \leq 0, \quad \forall j$

$\sum_{r=1}^{R} \tilde{y}_{r j} \mu_{r}-\sum_{d=1}^{D} \tilde{z}_{d j} \gamma_{d}-\sum_{h=1}^{H} \tilde{g}_{h j} \pi_{h} \leq 0, \quad \forall j$

$\tau_{i}, \omega_{k}, \gamma_{d}, \pi_{h}, \mu_{r} \geq 0, \quad \forall i, k, d, h, r$

$\tilde{\Psi}_{p}^{\text {Stagel }}=\operatorname{Max} \Omega$

S.t. $\quad \sum_{k=1}^{K} \tilde{f}_{k p} \omega_{k}+\sum_{d=1}^{D} \tilde{z}_{d p} \gamma_{d} \geq \Omega$

$\sum_{i=1}^{I} \tilde{x}_{i p} \tau_{i} \leq 1$ 


$$
\begin{aligned}
& \sum_{k=1}^{K} \tilde{f}_{k j} \omega_{k}+\sum_{d=1}^{D} \tilde{z}_{d j} \gamma_{d}-\sum_{i=1}^{I} \tilde{x}_{i j} \tau_{i} \leq 0, \quad \forall j \\
& \sum_{r=1}^{R} \tilde{y}_{r j} \mu_{r}-\sum_{d=1}^{D} \tilde{z}_{d j} \gamma_{d}-\sum_{h=1}^{H} \tilde{g}_{h j} \pi_{h} \leq 0, \quad \forall j \\
& \sum_{k=1}^{K} \tilde{f}_{k p} \omega_{k}+\sum_{d=1}^{D} \tilde{z}_{d p} \gamma_{d}+\sum_{r=1}^{R} \tilde{y}_{r p} \mu_{r}-\Psi_{p}^{\text {Overall }{ }^{*}} \sum_{d=1}^{D} \tilde{z}_{d p} \gamma_{d}-\Psi_{p}^{\text {Overall }} \sum_{h=1}^{H} \tilde{g}_{h p} \pi_{h} \geq \Psi_{p}^{\text {Overall* }} \\
& \tau_{i}, \omega_{k}, \gamma_{d}, \pi_{h}, \mu_{r} \geq 0, \quad \forall i, k, d, h, r
\end{aligned}
$$

As can be seen in the Models (12) and (13), there have been changes in the objective function and in equality constraints. But, none of these transformations changes the optimal solutions of the models (Peykani et al., 2018). Now, in order to dealing with imprecise and vague data in Models (12) and (13), adjustable possibilistic programming and chance-constrained programming will be applied.

It should be explained that the possibility theory introduced by Zadeh (1978) is a mathematical theory for dealing with certain types of uncertainty and it is an alternative to probability theory. In other words, the possibility approach is an applicable method to deal with the uncertainty caused by the absence or lack of knowledge about the exact value of model parameters in fuzzy mathematical programming (Peykani et al., 2018). Also, the chance-constrained programming is proposed by Charnes \& Cooper (1959) for the first time and it is wieldy employed as a powerful technique to solve optimization problems under probabilistic uncertainty (Shiraz et al., 2017).

It should be noted that in fuzzy linear programming models, fuzzy coefficients can be viewed as fuzzy variables and constraints can be considered as fuzzy events. Hence, the possibilities of fuzzy constraints can be determined by possibility theory. Recently, the novel fuzzy measure that is called general fuzzy (GF) measure is applied in order to measure the chances of occurrence of fuzzy events. In the GF measure, the attitude of a decision maker (DM) can be adjusted by setting the optimistic-pessimistic parameter (Peykani et al., 2019).

Let $\tilde{\psi}$ and $\tilde{\eta}$ be a trapezoidal fuzzy numbers that is determined by $\tilde{\psi}\left(\psi_{1}, \psi_{2}, \psi_{3}, \psi_{4}\right)$ and $\tilde{\eta}\left(\eta_{1}, \eta_{2}, \eta_{3}, \eta_{4}\right)$ with condition of $\psi_{1}<\psi_{2}<\psi_{3}<\psi_{4}$ and $\eta_{1}<\eta_{2}<\eta_{3}<\eta_{4}$ on the possibility space $(\xi, P(\xi), P o s)$ and $\phi$ be a crisp number. 
An equivalent crisp of fuzzy events $\{\tilde{\psi} \leq \phi\},\{\tilde{\psi} \geq \phi\},\{\tilde{\psi} \leq \tilde{\eta}\}$, and $\{\tilde{\psi} \geq \tilde{\eta}\}$ based on the general fuzzy measure are as Equations (14) to (17), respectively. Please note that $\alpha(0 \leq \alpha \leq 1)$ is confidence level for satisfying the constraints and $\beta(0 \leq \beta \leq 1)$ is optimistic-pessimistic parameter for adjusting the attitude of DM (Xu \& Zhou, 2011; Peykani et al., 2019).

$$
\begin{aligned}
& G F\{\tilde{\psi} \leq \phi\} \geq \alpha \Leftrightarrow\left\{\begin{array}{l}
\left(\frac{\beta-\alpha}{\beta}\right) \psi_{1}+\left(\frac{\alpha}{\beta}\right) \psi_{2} \leq \phi, \text { if } \alpha \leq \beta ; \\
\left(\frac{1-\alpha}{1-\beta}\right) \psi_{3}+\left(\frac{\alpha-\beta}{1-\beta}\right) \psi_{4} \leq \phi, \quad \text { if } \alpha>\beta .
\end{array}\right. \\
& G F\{\tilde{\psi} \geq \phi\} \geq \alpha \Leftrightarrow\left\{\begin{array}{l}
\left(\frac{\alpha}{\beta}\right) \psi_{3}+\left(\frac{\beta-\alpha}{\beta}\right) \psi_{4} \geq \phi, \text { if } \alpha \leq \beta ; \\
\left(\frac{\alpha-\beta}{1-\beta}\right) \psi_{1}+\left(\frac{1-\alpha}{1-\beta}\right) \psi_{2} \geq \phi, \quad \text { if } \alpha>\beta .
\end{array}\right. \\
& G F\{\tilde{\psi} \leq \tilde{\eta}\} \geq \alpha \Leftrightarrow\left\{\begin{array}{l}
\left(\frac{\beta-\alpha}{\beta}\right) \psi_{1}+\left(\frac{\alpha}{\beta}\right) \psi_{2} \leq\left(\frac{\alpha}{\beta}\right) \eta_{3}+\left(\frac{\beta-\alpha}{\beta}\right) \eta_{4}, \quad \text { if } \alpha \leq \beta ; \\
\left(\frac{1-\alpha}{1-\beta}\right) \psi_{3}+\left(\frac{\alpha-\beta}{1-\beta}\right) \psi_{4} \leq\left(\frac{\alpha-\beta}{1-\beta}\right) \eta_{1}+\left(\frac{1-\alpha}{1-\beta}\right) \eta_{2}, \quad \text { if } \alpha>\beta .
\end{array}\right. \\
& G F\{\tilde{\psi} \geq \tilde{\eta}\} \geq \alpha \Leftrightarrow\left\{\begin{array}{l}
\left(\frac{\alpha}{\beta}\right) \psi_{3}+\left(\frac{\beta-\alpha}{\beta}\right) \psi_{4} \geq\left(\frac{\beta-\alpha}{\beta}\right) \eta_{1}+\left(\frac{\alpha}{\beta}\right) \eta_{2}, \quad \text { if } \alpha \leq \beta ; \\
\left(\frac{\alpha-\beta}{1-\beta}\right) \psi_{1}+\left(\frac{1-\alpha}{1-\beta}\right) \psi_{2} \geq\left(\frac{1-\alpha}{1-\beta}\right) \eta_{3}+\left(\frac{\alpha-\beta}{1-\beta}\right) \eta_{4}, \quad \text { if } \alpha>\beta .
\end{array}\right.
\end{aligned}
$$

It is important to stress that in the GF measure, $\beta=0$ and $\beta=1$ indicate that the DM takes pessimistic and optimistic viewpoint, respectively. Now, the APP based on GF measure as well as CCP are used to deal with imprecise and vague data in fuzzy chance constraints of FNDEA models and converting them to their equivalent crisp. According to GF measure and CCP, Models (12) and (13) are defined as Models (18) and (19), respectively: 
S.t. $\quad G F\left\{\sum_{k=1}^{K} \tilde{f}_{k p} \omega_{k}+\sum_{d=1}^{D} \tilde{z}_{d p} \gamma_{d}+\sum_{r=1}^{R} \tilde{y}_{r p} \mu_{r} \geq \Omega\right\} \geq \alpha$

$G F\left\{\sum_{i=1}^{L} \tilde{x}_{i p} \tau_{i}+\sum_{d=1}^{D} \tilde{z}_{d p} \gamma_{d}+\sum_{h=1}^{H} \tilde{g}_{h p} \pi_{h} \leq 1\right\} \geq \alpha$

$G F\left\{\sum_{k=1}^{K} \tilde{f}_{k j} \omega_{k}+\sum_{d=1}^{D} \tilde{z}_{d j} \gamma_{d}-\sum_{i=1}^{L} \tilde{x}_{i j} \tau_{i} \leq 0\right\} \geq \alpha, \quad \forall j$

$G F\left\{\sum_{r=1}^{R} \tilde{y}_{r j} \mu_{r}-\sum_{d=1}^{D} \tilde{z}_{d j} \gamma_{d}-\sum_{h=1}^{H} \tilde{g}_{h j} \pi_{h} \leq 0\right\} \geq \alpha, \quad \forall j$

$\tau_{i}, \omega_{k}, \gamma_{d}, \pi_{h}, \mu_{r} \geq 0, \quad \forall i, k, d, h, r$

$\tilde{\Psi}_{p}^{\text {Stagel }}=\operatorname{Max} \Omega$

(19)

S.t. $\quad G F\left\{\sum_{k=1}^{K} \tilde{f}_{k p} \omega_{k}+\sum_{d=1}^{D} \tilde{z}_{d p} \gamma_{d} \geq \Omega\right\} \geq \alpha$

$G F\left\{\sum_{i=1}^{L} \tilde{x}_{i p} \tau_{i} \leq 1\right\} \geq \alpha$

$G F\left\{\sum_{k=1}^{K} \tilde{f}_{k j} \omega_{k}+\sum_{d=1}^{D} \tilde{z}_{d j} \gamma_{d}-\sum_{i=1}^{L} \tilde{x}_{i j} \tau_{i} \leq 0\right\} \geq \alpha, \quad \forall j$

$G F\left\{\sum_{r=1}^{R} \tilde{y}_{r j} \mu_{r}-\sum_{d=1}^{D} \tilde{z}_{d j} \gamma_{d}-\sum_{h=1}^{H} \tilde{g}_{h j} \pi_{h} \leq 0\right\} \geq \alpha, \quad \forall j$

$G F\left\{\sum_{k=1}^{K} \tilde{f}_{k p} \omega_{k}+\sum_{d=1}^{D} \tilde{z}_{d p} \gamma_{d}+\sum_{r=1}^{R} \tilde{y}_{r p} \mu_{r}\right.$

$\left.-\Psi_{p}^{\text {Overall }} \sum_{d=1}^{D} \tilde{z}_{d p} \gamma_{d}-\Psi_{p}^{\text {Overal } *} \sum_{h=1}^{H} \tilde{g}_{h p} \pi_{h} \geq \Psi_{p}^{\text {Overall* }}\right\} \geq \alpha$

$\tau_{i}, \omega_{k}, \gamma_{d}, \pi_{h}, \mu_{r} \geq 0, \quad \forall i, k, d, h, r$

As it can be seen in Equations (14) to (17), for the confidence level ( $\alpha$ ) greater or less than optimistic-pessimistic parameter $(\beta)$, an equivalent crisp of fuzzy chance constraints is different. Conceptually, the novel FNDEA model to calculate of overall efficiency score of $\mathrm{DMU}_{p}$ under fuzzy data for $\alpha \leq \beta$ and $\alpha>\beta$ are presented as Models (20) and (21), respectively: 


$$
\begin{array}{ll}
\text { S.t. } & \sum_{k=1}^{K}\left(\left(\frac{\alpha}{\beta}\right) f_{k p}^{3}+\left(\frac{\beta-\alpha}{\beta}\right) f_{k p}^{4}\right) \omega_{k}+\sum_{d=1}^{D}\left(\left(\frac{\alpha}{\beta}\right) z_{d p}^{3}+\left(\frac{\beta-\alpha}{\beta}\right) z_{d p}^{4}\right) \gamma_{d} \\
& +\sum_{r=1}^{R}\left(\left(\frac{\alpha}{\beta}\right) y_{r p}^{3}+\left(\frac{\beta-\alpha}{\beta}\right) y_{r p}^{4}\right) \mu_{r} \geq \Omega \\
& \sum_{i=1}^{L}\left(\left(\frac{\beta-\alpha}{\beta}\right) x_{i p}^{1}+\left(\frac{\alpha}{\beta}\right) x_{i p}^{2}\right) \tau_{i}+\sum_{d=1}^{D}\left(\left(\frac{\beta-\alpha}{\beta}\right) z_{d p}^{1}+\left(\frac{\alpha}{\beta}\right) z_{d p}^{2}\right) \gamma_{d} \\
+ & \sum_{h=1}^{H}\left(\left(\frac{\beta-\alpha}{\beta}\right) g_{h p}^{1}+\left(\frac{\alpha}{\beta}\right) g_{h p}^{2}\right) \pi_{h} \leq 1 \\
& \sum_{k=1}^{K}\left(\left(\frac{\beta-\alpha}{\beta}\right) f_{k j}^{1}+\left(\frac{\alpha}{\beta}\right) f_{k j}^{2}\right) \omega_{k}+\sum_{d=1}^{D}\left(\left(\frac{\beta-\alpha}{\beta}\right) z_{d j}^{1}+\left(\frac{\alpha}{\beta}\right) z_{d j}^{2}\right) \gamma_{d} \\
- & \sum_{i=1}^{L}\left(\left(\frac{\alpha}{\beta}\right) x_{i j}^{3}+\left(\frac{\beta-\alpha}{\beta}\right) x_{i j}^{4}\right) \tau_{i} \leq 0, \quad \forall j \\
& \sum_{r=1}^{R}\left(\left(\frac{\beta-\alpha}{\beta}\right) y_{r j}^{1}+\left(\frac{\alpha}{\beta}\right) y_{r j}^{2}\right) \mu_{r}-\sum_{d=1}^{D}\left(\left(\frac{\alpha}{\beta}\right) z_{d j}^{3}+\left(\frac{\beta-\alpha}{\beta}\right) z_{d j}^{4}\right) \gamma_{d} \\
& -\sum_{h=1}^{H}\left(\left(\frac{\alpha}{\beta}\right) g_{h j}^{3}+\left(\frac{\beta-\alpha}{\beta}\right) g_{h j}^{4}\right) \pi_{h} \leq 0, \forall j \\
& \tau_{i}, \omega_{k}, \gamma_{d}, \pi_{h}, \mu_{r} \geq 0, \forall i, k, d, h, r
\end{array}
$$

$\Psi_{p(\alpha>\beta)}^{\text {Overall }}=\operatorname{Max} \Omega$

S.t. $\quad \sum_{k=1}^{K}\left(\left(\frac{\alpha-\beta}{1-\beta}\right) f_{k p}^{1}+\left(\frac{1-\alpha}{1-\beta}\right) f_{k p}^{2}\right) \omega_{k}+\sum_{d=1}^{D}\left(\left(\frac{\alpha-\beta}{1-\beta}\right) z_{d p}^{1}+\left(\frac{1-\alpha}{1-\beta}\right) z_{d p}^{2}\right) \gamma_{d}$

$+\sum_{r=1}^{R}\left(\left(\frac{\alpha-\beta}{1-\beta}\right) y_{r p}^{1}+\left(\frac{1-\alpha}{1-\beta}\right) y_{r p}^{2}\right) \mu_{r} \geq \Omega$

$\sum_{i=1}^{L}\left(\left(\frac{1-\alpha}{1-\beta}\right) x_{i p}^{3}+\left(\frac{\alpha-\beta}{1-\beta}\right) x_{i p}^{4}\right) \tau_{i}+\sum_{d=1}^{D}\left(\left(\frac{1-\alpha}{1-\beta}\right) z_{d p}^{3}+\left(\frac{\alpha-\beta}{1-\beta}\right) z_{d p}^{4}\right) \gamma_{d}$

$+\sum_{h=1}^{H}\left(\left(\frac{1-\alpha}{1-\beta}\right) g_{h p}^{3}+\left(\frac{\alpha-\beta}{1-\beta}\right) g_{h p}^{4}\right) \pi_{h} \leq 1$

$\sum_{k=1}^{K}\left(\left(\frac{1-\alpha}{1-\beta}\right) f_{k j}^{3}+\left(\frac{\alpha-\beta}{1-\beta}\right) f_{k j}^{4}\right) \omega_{k}+\sum_{d=1}^{D}\left(\left(\frac{1-\alpha}{1-\beta}\right) z_{d j}^{3}+\left(\frac{\alpha-\beta}{1-\beta}\right) z_{d j}^{4}\right) \gamma_{d}$

$-\sum_{i=1}^{L}\left(\left(\frac{\alpha-\beta}{1-\beta}\right) x_{i j}^{1}+\left(\frac{1-\alpha}{1-\beta}\right) x_{i j}^{2}\right) \tau_{i} \leq 0, \quad \forall j$ 


$$
\begin{aligned}
& \sum_{r=1}^{R}\left(\left(\frac{1-\alpha}{1-\beta}\right) y_{r j}^{3}+\left(\frac{\alpha-\beta}{1-\beta}\right) y_{r j}^{4}\right) \mu_{r}-\sum_{d=1}^{D}\left(\left(\frac{\alpha-\beta}{1-\beta}\right) z_{d j}^{1}+\left(\frac{1-\alpha}{1-\beta}\right) z_{d j}^{2}\right) \gamma_{d} \\
- & \sum_{h=1}^{H}\left(\left(\frac{\alpha-\beta}{1-\beta}\right) g_{h j}^{1}+\left(\frac{1-\alpha}{1-\beta}\right) g_{h j}^{2}\right) \pi_{h} \leq 0, \quad \forall j \\
& \tau_{i}, \omega_{k}, \gamma_{d}, \pi_{h}, \mu_{r} \geq 0, \quad \forall i, k, d, h, r
\end{aligned}
$$

Then, the efficiency of the first stage in the presence of fuzzy data for $\alpha \leq \beta$ and $\alpha>\beta$ are estimated by solving Models (22) and (23) while $\Psi_{p(\alpha \leq \beta)}^{\text {Overal* }}$ as well as $\Psi_{p(\alpha>\beta)}^{\text {Overall* }}$ are obtained from Models (20) and (21), respectively:

$\Psi_{p(\alpha \leq \beta)}^{\text {Stagel }}=\operatorname{Max} \Omega$

S.t. $\quad \sum_{k=1}^{K}\left(\left(\frac{\alpha}{\beta}\right) f_{k p}^{3}+\left(\frac{\beta-\alpha}{\beta}\right) f_{k p}^{4}\right) \omega_{k}$

$+\sum_{d=1}^{D}\left(\left(\frac{\alpha}{\beta}\right) z_{d p}^{3}+\left(\frac{\beta-\alpha}{\beta}\right) z_{d p}^{4}\right) \gamma_{d} \geq \Omega$

$\sum_{i=1}^{L}\left(\left(\frac{\beta-\alpha}{\beta}\right) x_{i p}^{1}+\left(\frac{\alpha}{\beta}\right) x_{i p}^{2}\right) \tau_{i} \leq 1$

$\sum_{k=1}^{K}\left(\left(\frac{\beta-\alpha}{\beta}\right) f_{k j}^{1}+\left(\frac{\alpha}{\beta}\right) f_{k j}^{2}\right) \omega_{k}+\sum_{d=1}^{D}\left(\left(\frac{\beta-\alpha}{\beta}\right) z_{d j}^{1}+\left(\frac{\alpha}{\beta}\right) z_{d j}^{2}\right) \gamma_{d}$

$-\sum_{i=1}^{L}\left(\left(\frac{\alpha}{\beta}\right) x_{i j}^{3}+\left(\frac{\beta-\alpha}{\beta}\right) x_{i j}^{4}\right) \tau_{i} \leq 0, \quad \forall j$

$\sum_{r=1}^{R}\left(\left(\frac{\beta-\alpha}{\beta}\right) y_{r j}^{1}+\left(\frac{\alpha}{\beta}\right) y_{r j}^{2}\right) \mu_{r}-\sum_{d=1}^{D}\left(\left(\frac{\alpha}{\beta}\right) z_{d j}^{3}+\left(\frac{\beta-\alpha}{\beta}\right) z_{d j}^{4}\right) \gamma_{d}$

$-\sum_{h=1}^{H}\left(\left(\frac{\alpha}{\beta}\right) g_{h j}^{3}+\left(\frac{\beta-\alpha}{\beta}\right) g_{h j}^{4}\right) \pi_{h} \leq 0, \quad \forall j$

$\sum_{k=1}^{K}\left(\left(\frac{\alpha}{\beta}\right) f_{k p}^{3}+\left(\frac{\beta-\alpha}{\beta}\right) f_{k p}^{4}\right) \omega_{k}+\sum_{d=1}^{D}\left(\left(\frac{\alpha}{\beta}\right) z_{d p}^{3}+\left(\frac{\beta-\alpha}{\beta}\right) z_{d p}^{4}\right) \gamma_{d}$

$+\sum_{r=1}^{R}\left(\left(\frac{\alpha}{\beta}\right) y_{r p}^{3}+\left(\frac{\beta-\alpha}{\beta}\right) y_{r p}^{4}\right) \mu_{r}-\Psi_{p(\alpha, \beta)}^{\text {Overall* }} \sum_{d=1}^{D}\left(\left(\frac{\beta-\alpha}{\beta}\right) z_{d p}^{1}+\left(\frac{\alpha}{\beta}\right) z_{d p}^{2}\right) \gamma_{d}$

$-\Psi_{p(\alpha, \beta)}^{\text {Overall* }} \sum_{h=1}^{H}\left(\left(\frac{\beta-\alpha}{\beta}\right) g_{h p}^{1}+\left(\frac{\alpha}{\beta}\right) g_{h p}^{2}\right) \pi_{h} \geq \Psi_{p(\alpha, \beta)}^{\text {Overall* }}$

$\tau_{i}, \omega_{k}, \gamma_{d}, \pi_{h}, \mu_{r} \geq 0, \quad \forall i, k, d, h, r$ 
$\Psi_{p(\alpha>\beta)}^{\text {Stagel }}=\operatorname{Max} \Omega$

S.t. $\quad \sum_{k=1}^{K}\left(\left(\frac{\alpha-\beta}{1-\beta}\right) f_{k p}^{1}+\left(\frac{1-\alpha}{1-\beta}\right) f_{k p}^{2}\right) \omega_{k}$

$+\sum_{d=1}^{D}\left(\left(\frac{\alpha-\beta}{1-\beta}\right) z_{d p}^{1}+\left(\frac{1-\alpha}{1-\beta}\right) z_{d p}^{2}\right) \gamma_{d} \geq \Omega$

$\sum_{i=1}^{L}\left(\left(\frac{1-\alpha}{1-\beta}\right) x_{i p}^{3}+\left(\frac{\alpha-\beta}{1-\beta}\right) x_{i p}^{4}\right) \tau_{i} \leq 1$

$\sum_{k=1}^{K}\left(\left(\frac{1-\alpha}{1-\beta}\right) f_{k j}^{3}+\left(\frac{\alpha-\beta}{1-\beta}\right) f_{k j}^{4}\right) \omega_{k}+\sum_{d=1}^{D}\left(\left(\frac{1-\alpha}{1-\beta}\right) z_{d j}^{3}+\left(\frac{\alpha-\beta}{1-\beta}\right) z_{d j}^{4}\right) \gamma_{d}$

$-\sum_{i=1}^{L}\left(\left(\frac{\alpha-\beta}{1-\beta}\right) x_{i j}^{1}+\left(\frac{1-\alpha}{1-\beta}\right) x_{i j}^{2}\right) \tau_{i} \leq 0, \quad \forall j$

$\sum_{r=1}^{R}\left(\left(\frac{1-\alpha}{1-\beta}\right) y_{r j}^{3}+\left(\frac{\alpha-\beta}{1-\beta}\right) y_{r j}^{4}\right) \mu_{r}-\sum_{d=1}^{D}\left(\left(\frac{\alpha-\beta}{1-\beta}\right) z_{d j}^{1}+\left(\frac{1-\alpha}{1-\beta}\right) z_{d j}^{2}\right) \gamma_{d}$

$-\sum_{h=1}^{H}\left(\left(\frac{\alpha-\beta}{1-\beta}\right) g_{h j}^{1}+\left(\frac{1-\alpha}{1-\beta}\right) g_{h j}^{2}\right) \pi_{h} \leq 0, \quad \forall j$

$\sum_{k=1}^{K}\left(\left(\frac{\alpha-\beta}{1-\beta}\right) f_{k p}^{1}+\left(\frac{1-\alpha}{1-\beta}\right) f_{k p}^{2}\right) \omega_{k}+\sum_{d=1}^{D}\left(\left(\frac{\alpha-\beta}{1-\beta}\right) z_{d p}^{1}+\left(\frac{1-\alpha}{1-\beta}\right) z_{d p}^{2}\right) \gamma_{d}$

$+\sum_{r=1}^{R}\left(\left(\frac{\alpha-\beta}{1-\beta}\right) y_{r p}^{1}+\left(\frac{1-\alpha}{1-\beta}\right) y_{r p}^{2}\right) \mu_{r}-\Psi_{p(\alpha, \beta)}^{\text {Overall* }} \sum_{d=1}^{D}\left(\left(\frac{1-\alpha}{1-\beta}\right) z_{d p}^{3}+\left(\frac{\alpha-\beta}{1-\beta}\right) z_{d p}^{4}\right) \gamma_{d}$

$-\Psi_{p(\alpha, \beta)}^{\text {Overall } *} \sum_{h=1}^{H}\left(\left(\frac{1-\alpha}{1-\beta}\right) g_{h p}^{3}+\left(\frac{\alpha-\beta}{1-\beta}\right) g_{h p}^{4}\right) \pi_{h} \geq \underset{p(\alpha, \beta)}{\Psi_{\text {Overall* }}^{\text {Ov }}}$

$\tau_{i}, \omega_{k}, \gamma_{d}, \pi_{h}, \mu_{r} \geq 0, \quad \forall i, k, d, h, r$

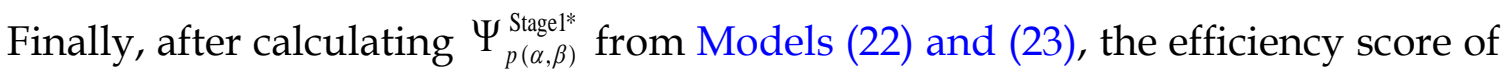
stage 2 is estimated using Equation (24):

$\Psi_{p(\alpha, \beta)}^{\text {Stage2* }}=\frac{\Psi_{p}^{\text {Overall }_{(\alpha, \beta)}}-\xi_{1(\alpha, \beta)}^{*} \Psi_{p(\alpha, \beta)}^{\text {Stage } 1^{*}}}{\xi_{2(\alpha, \beta)}^{*}}$

It should be noted that using a binary variable and a sufficient big number, the linearization of incompatible constraints and the integration of FNDEA models for $\alpha \leq \beta$ and $\alpha>\beta$ can be made. For more details, integrated framework of FNDEA models are presented in Appendix B. 
It is worth noting that the efficiency scores obtained from proposed AFNDEA approach can be greater than one. By inspiring of Peykani et al. (2019), DMUs can be classified as follows:

- DMU is ultra-efficient if its efficiency scores $\Psi^{*} \geq 1$ for all confidence levels.

- DMU is marginal-efficient if its efficiency scores $\Psi^{*} \geq 1$ for some of confidence levels.

- DMU is inefficient if its efficiency scores $\Psi^{*}<1$ for all confidence levels.

At the end of this section, the process of performance appraisal of two-stage DMUs based on proposed adjustable fuzzy network data envelopment analysis (AFNDEA) approach can be summarized as follows: Firstly, confidence level $(\alpha)$ and optimistic-pessimistic parameter $(\beta)$ should be determined by the DM. Then, Models (20) and (21) should be solved for desired value of these parameters $(\alpha, \beta)$ and the overall efficiency score of $\mathrm{DMU}_{p}\left(\Psi_{p(\alpha, \beta)}^{\text {Overall* }}\right)$ is calculated. Next step, the efficiency score of first stage $\left(\Psi_{p(\alpha, \beta)}^{\text {Stagel*}}\right)$ is estimated by solving Models (22) and (23) according to desired value of these parameters $(\alpha, \beta)$ and $\Psi_{p(\alpha, \beta)}^{\text {Overal* }}$. Finally, the efficiency score of second stage $\left(\Psi_{p(\alpha, \beta)}^{\text {Stage }^{*}}\right)$ is calculated using Equation (24). Notably, if assumed that the stage 2 is more important, the efficiency of stages 2 and 1 will be estimated in a similar manner that is introduced in Appendix C.

\section{A New Ranking Method for Two-Stage DMUs under Fuzzy Data}

Proposing a full ranking method to rank all the DMUs is one of the important issues in DEA literature. In this section, the novel ranking method for two-stage DMUs under fuzzy environment will be presented using proposed AFNDEA approach. As explained in the previous section, the AFNDEA approach is capable to calculate the efficiency score of two-stage DMUs for all possible states of confidence level $(\alpha)$ and optimistic-pessimistic parameter $(\beta)$. Figure 5 shows the three-dimensional (3D) presentation of efficiency scores obtained from AFNDEA approach for all pair of $(\alpha, \beta)$. As it can be seen in Figure 5, if all possible efficiency values from the 
minimum to the maximum value are calculated for the DMUs and then plotted, the efficiency surface is obtained. Accordingly, the volume enclosed under the efficiency surface can be considered as a ranking criterion. In other words, all the DMUs can be ranked based on the amount of volume of gray area that shown in Figure 5.

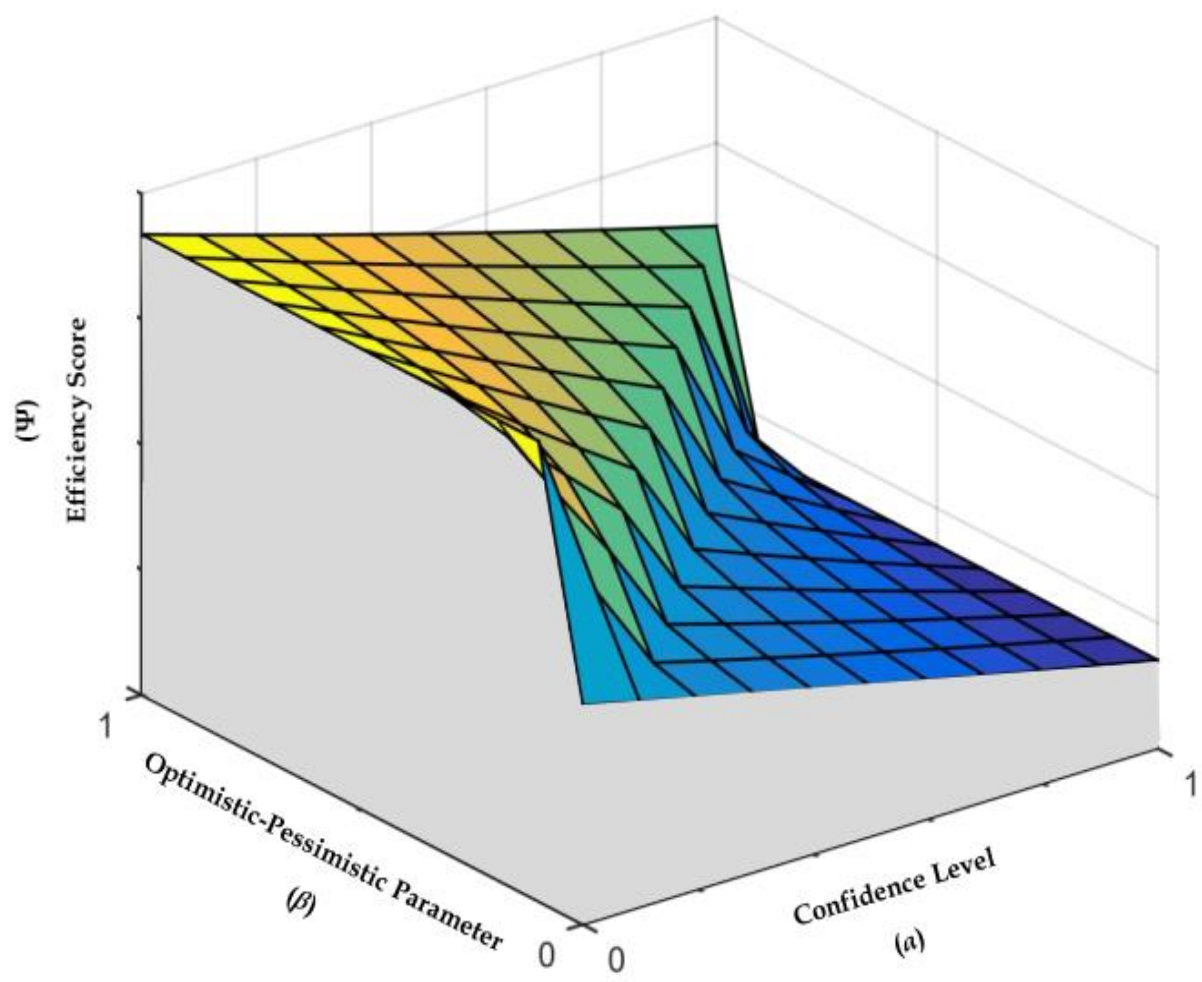

Figure 5. The Efficiency Surface Based on AFNDEA Approach

In order to calculate the volume enclosed under the efficiency surface (gray area), the triple integral can be applied. But, due to the function of efficiency surface is not specified, the triple integral cannot be used. As a result, the heuristic approach is presented in following for estimating the volume of gray area.

Note that the range of confidence level $(0 \leq \alpha \leq 1)$ and optimistic-pessimistic parameter $(0 \leq \beta \leq 1)$. are clear and the floor area is 1 . Also, only the height of the 3D shape (efficiency score) differs at different points. Therefore, in order to estimate the volume of gray area, it is simply necessary to calculate the average of the efficiency values at different points of $(\alpha, \beta)$.

Accordingly, assume that $\delta_{\alpha}$ and $\delta_{\beta}$ denote to the number of confidence levels and optimistic-pessimistic attitudes, respectively, that considered by DM for solving 
AFNDEA approach. The volume enclosed under the efficiency surface for overall, stage 1, and stage 2 of network DMUs can be estimated using Equations (25) to (27), respectively:

$$
\begin{aligned}
& \Omega^{\text {Overall }}=\frac{\sum_{\alpha=0}^{1} \sum_{\beta=0}^{1} \Psi_{p(\alpha, \beta)}^{\text {Overall* }}}{\delta_{\alpha} \delta_{\beta}} \\
& \Omega^{\text {Stage1 }}=\frac{\sum_{\alpha=0}^{1} \sum_{\beta=0}^{1} \Psi_{p(\alpha, \beta)}^{\text {Stage } *}}{\delta_{\alpha} \delta_{\beta}} \\
& \Omega^{\text {Stage2 }}=\frac{\sum_{\alpha=0}^{1} \sum_{\beta=0}^{1} \Psi_{p(\alpha, \beta)}^{\text {Stage } 2^{*}}}{\delta_{\alpha} \delta_{\beta}}
\end{aligned}
$$

It must be emphasized that whatever the $\delta_{\alpha}$ and $\delta_{\beta}$ are more, the accuracy of the ranking method increases.

Finally, the presented ranking method for network DMUs with two-stage structure under fuzzy environment can be summarized as follows:

Step 1: Calculating the overall efficiency score $\left(\Psi_{p(\alpha, \beta)}^{\text {Overal* }}\right)$ using Models (20) and (21) for all optimistic-pessimistic attitudes and confidence levels.

Step 2: Plotting all the results obtained from AFNDEA approach for all pair of $(\alpha, \beta)$ and drawing the surface of all efficiency scores.

Step 3: Estimating the volume of three-dimensional shape in below the efficiency surface $\left(\Omega^{\text {Overall }}\right)$ using Equation (25).

Step 4: Ranking of DMUs based on amount of volume as a ranking criterion from highest to lowest value.

Notably, in a similar manner for ranking of sub-DMUs (stages 1 and 2), in the first step of proposed algorithm, the efficiency score of first stage $\left(\Psi_{p(\alpha, \beta)}^{\text {Stage }\left.\right|^{*}}\right)$ and second stage $\left(\Psi_{p(\alpha, \beta)}^{\text {Stage }^{*}}\right)$ should be calculated using Models (22) and (23) as well as Equation (24), respectively. Moreover, in the third step, the volume enclosed under efficiency 
surface of stage $1\left(\Omega^{\text {Stagel }}\right)$ and stage $2\left(\Omega^{\text {Stage2 }}\right)$, can be estimated using Equations $(26)$ and (27), respectively.

\section{Real-Life Application: Investment Firms}

In this section the proposed adjustable fuzzy network DEA approach as well as presented ranking method will be applied for measuring performance of investment firms from Iranian financial market. Investment firms such as mutual funds have a significant role in financial markets. IFs invest the money received from investors on a specific investment plan and each investor will be shared in the returns and risks of investment in proportion to his/her interest in the IFs.

Premachandra et al. (2012) were the pioneer researchers that used two-stage DEA approach in order to assess the performance of mutual fund families by considering their operational and portfolio management processes. Then, Galagedera et al. (2016) developed the suggested two-stage structure for performance appraisal of MFs by adding the total cash flow of investors as a leakage variable in operational management stage.

Galagedera et al. (2018) proposed a new structure for network DEA method to MFs performance appraisal considering MF structure as a three-stage process including operational, resource, and portfolio management. Last but not the least, Galagedera (2019) introduced two-stage DEA model with non-discretionary output in operational management stage for modelling social responsibility in MF performance evaluation.

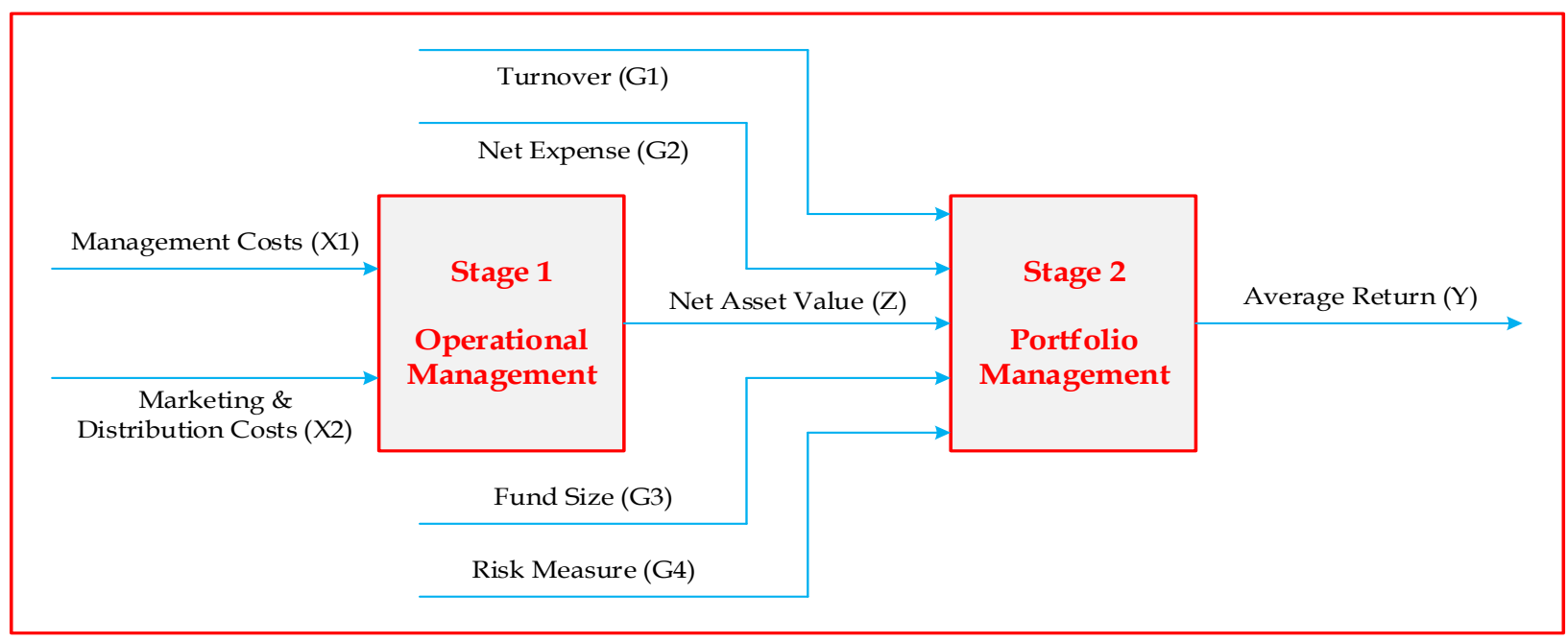


Figure 6. The Structure of Investment Firms

As shown in Figure 6, the overall efficiency of the investment firm can be decomposed into two stages (processes) where first stage denotes the operational management function and second stage denotes the portfolio management function (Premachandra et al., 2012). A brief explanation of inputs (X), intermediate factor (Z), additional inputs $(\mathrm{G})$, and output $(\mathrm{Y})$ of IFs structure are introduced as follows:

Management Costs (X1): Fees paid to investment advisors. Marketing \& Distribution Costs (X2): Cost of marketing and selling IF shares. Net Asset Value (Z): Total value of portfolio less liabilities in base currency. Turnover (G1): Percentage of holdings replaced. Net Expense (G2): Annual fee expressed as a percentage to cover expenses such as administrative fees, operating costs and all other asset-based costs incurred by the fund. Fund Size (G3): Market value of portfolio in base currency. Risk: Measure (G4) Standard deviation (SD) of monthly return. Average Return (Y): The money made or lost by an IF over time.

According to Figure 6, the financial data set for 5 mutual funds in Iranian financial market are extracted from March 2015 to March 2017. Then, by inspiring the idea of Kao \& Liu (2011) and experts' opinions, trapezoidal fuzzy numbers are constructed for X1, X2, Z, G1, G2, G3, G4, and Y, as shown in Table 2.

Table 2. Fuzzy Data Set for 5 Mutual Funds in Iran

\begin{tabular}{|c|c|c|c|c|c|}
\hline Index & MF 01 & MF 02 & MF 03 & MF 04 & MF 05 \\
\hline X1 & $\begin{array}{l}(0.1771,0.1846 \\
0.1883,0.1958)\end{array}$ & $\begin{array}{l}(1.4784,1.5406, \\
1.5718,1.6340)\end{array}$ & $\begin{array}{l}(0.8007,0.8344 \\
0.8512,0.8849)\end{array}$ & $\begin{array}{l}(4.2664,4.4460, \\
4.5358,4.7155)\end{array}$ & $\begin{array}{l}(0.2254,0.2349, \\
0.2397,0.2492)\end{array}$ \\
\hline $\mathrm{X} 2$ & $\begin{array}{c}(166.2184,173.2171 \\
176.7164,183.7151)\end{array}$ & $\begin{array}{l}(5.0883,5.3025 \\
5.4097,5.6239)\end{array}$ & $\begin{array}{l}(78.8159,82.1345, \\
83.7938,87.1123)\end{array}$ & $\begin{array}{l}(0.0133,0.0138, \\
0.0141,0.0146)\end{array}$ & $\begin{array}{l}(0.0117,0.0122, \\
0.0125,0.0130)\end{array}$ \\
\hline $\mathbf{Z}$ & $\begin{array}{l}(2.5044,2.6098, \\
2.6625,2.7680)\end{array}$ & $\begin{array}{l}(9.8666,10.2821, \\
10.4898,10.9052)\end{array}$ & $\begin{array}{l}(4.1210,4.2945, \\
4.3813,4.5548)\end{array}$ & $\begin{array}{l}(15.3608,16.0075, \\
16.3309,16.9777)\end{array}$ & $\begin{array}{l}(2.3890,2.4896, \\
2.5398,2.6404)\end{array}$ \\
\hline G1 & $\begin{array}{l}(0.5933,0.6183, \\
0.6307,0.6557)\end{array}$ & $\begin{array}{l}(0.3277,0.3415, \\
0.3483,0.3621)\end{array}$ & $\begin{array}{l}(0.2732,0.2847 \\
0.2905,0.3020)\end{array}$ & $\begin{array}{l}(0.4124,0.4298 \\
0.4384,0.4558)\end{array}$ & $\begin{array}{l}(0.1816,0.1893, \\
0.1931,0.2008)\end{array}$ \\
\hline G2 & $\begin{array}{l}(3.2300,3.3660, \\
3.4340,3.5700)\end{array}$ & $\begin{array}{l}(4.6550,4.8510, \\
4.9490,5.1450)\end{array}$ & $\begin{array}{l}(4.2085,4.3857, \\
4.4743,4.6515)\end{array}$ & $\begin{array}{l}(4.2750,4.4550, \\
4.5450,4.7250)\end{array}$ & $\begin{array}{l}(8.7400,9.1080, \\
9.2920,9.6600)\end{array}$ \\
\hline G3 & $\begin{array}{l}(0.0116,0.0121, \\
0.0123,0.0128)\end{array}$ & $\begin{array}{l}(0.1334,0.1390, \\
0.1418,0.1474)\end{array}$ & $\begin{array}{l}(0.0858,0.0895 \\
0.0913,0.0949)\end{array}$ & $\begin{array}{l}(0.1750,0.1824, \\
0.1861,0.1934)\end{array}$ & $\begin{array}{l}(0.0121,0.0126, \\
0.0128,0.0134)\end{array}$ \\
\hline G4 & $\begin{array}{l}(1.1590,1.2078 \\
1.2322,1.2810)\end{array}$ & $\begin{array}{l}(0.9310,0.9702, \\
0.9898,1.0290)\end{array}$ & $\begin{array}{l}(2.4225,2.5245, \\
2.5755,2.6775)\end{array}$ & $\begin{array}{l}(2.0140,2.0988, \\
2.1412,2.2260)\end{array}$ & $\begin{array}{l}(2.3750,2.4750, \\
2.5250,2.6250)\end{array}$ \\
\hline
\end{tabular}




\begin{tabular}{cccccc}
\hline $\mathbf{Y}$ & $(55.5180,57.8556$, & $(37.9810,39.5802$, & $(23.5220,24.5124$, & $(49.4000,51.4800$, & $(5.2440,5.4648$, \\
& $59.0244,61.3620)$ & $40.3798,41.9790)$ & $25.0076,25.9980)$ & $52.5200,54.6000)$ & $5.5752,5.7960)$ \\
\hline
\end{tabular}

Please note that the convexity axiom is one of the fundamental assumptions of the production possibility set (PPS) and this axiom cannot be fully satisfied where data includes ratio variables (Emrouznejad \& Amin, 2009). In current study, since all selected mutual funds are about the same size, the convexity issue can be eliminated (Hanafizadeh et al. 2014). Now, after collecting data in trapezoidal membership function, the proposed adjustable fuzzy NDEA model will be run for different optimistic-pessimistic parameter and confidence levels. The results of efficiency scores for overall, stage 1, and stage 2 based on AFNDEA approach at different optimisticpessimistic parameters and confidence levels from 0 to 1 are presented in Tables 3 to 5 , respectively.

Table 3. The Overall Efficiency Scores of MFs Based on AFNDEA Approach

\begin{tabular}{|c|c|c|c|c|c|c|c|c|c|c|c|c|c|}
\hline \multirow{27}{*}{ 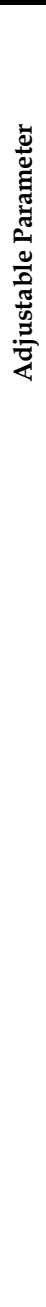 } & & \multicolumn{11}{|c|}{ Confidence Level } & \multirow{13}{*}{$\begin{array}{l}3 \\
\text { 帛 }\end{array}$} \\
\hline & & 0 & 0.1 & 0.2 & 0.3 & 0.4 & 0.5 & 0.6 & 0.7 & 0.8 & 0.9 & 1 & \\
\hline & 0 & 0.9048 & 0.8902 & 0.8758 & 0.8616 & 0.8477 & 0.8340 & 0.8205 & 0.8072 & 0.7941 & 0.7813 & 0.7686 & \\
\hline & 0.1 & 1.1554 & 0.9816 & 0.8886 & 0.8726 & 0.8570 & 0.8416 & 0.8265 & 0.8116 & 0.7970 & 0.7827 & 0.7686 & \\
\hline & 0.2 & 1.1554 & 1.0649 & 0.9816 & 0.8866 & 0.8687 & 0.8512 & 0.8340 & 0.8172 & 0.8007 & 0.7845 & 0.7686 & \\
\hline & 0.3 & 1.1554 & 1.0943 & 1.0364 & 0.9816 & 0.8840 & 0.8636 & 0.8438 & 0.8243 & 0.8053 & 0.7868 & 0.7686 & \\
\hline & 0.4 & 1.1554 & 1.1092 & 1.0649 & 1.0224 & 0.9816 & 0.8806 & 0.8570 & 0.8340 & 0.8116 & 0.7898 & 0.7686 & \\
\hline & 0.5 & 1.1554 & 1.1183 & 1.0824 & 1.0477 & 1.0141 & 0.9816 & 0.8758 & 0.8477 & 0.8205 & 0.7941 & 0.7686 & \\
\hline & 0.6 & 1.1554 & 1.1244 & 1.0943 & 1.0649 & 1.0364 & 1.0086 & 0.9816 & 0.8687 & 0.8340 & 0.8007 & 0.7686 & \\
\hline & 0.7 & 1.1554 & 1.1288 & 1.1028 & 1.0774 & 1.0526 & 1.0284 & 1.0047 & 0.9816 & 0.8570 & 0.8116 & 0.7686 & \\
\hline & 0.8 & 1.1554 & 1.1321 & 1.1092 & 1.0868 & 1.0649 & 1.0434 & 1.0224 & 1.0018 & 0.9816 & 0.8340 & 0.7686 & \\
\hline & 0.9 & 1.1554 & 1.1347 & 1.1143 & 1.0943 & 1.0746 & 1.0553 & 1.0364 & 1.0178 & 0.9995 & 0.9816 & 0.7686 & \\
\hline & 1 & 1.1554 & 1.1367 & 1.1183 & 1.1002 & 1.0824 & 1.0649 & 1.0477 & 1.0308 & 1.0141 & 0.9977 & 0.9816 & \\
\hline & 0 & 0.9499 & 0.9347 & 0.9198 & 0.9051 & 0.8906 & 0.8764 & 0.8624 & 0.8486 & 0.8350 & 0.8216 & 0.8085 & \multirow{11}{*}{$\begin{array}{l}3 \\
\text { T } \\
\text { N }\end{array}$} \\
\hline & 0.1 & 1.2089 & 1.0291 & 0.9330 & 0.9165 & 0.9002 & 0.8842 & 0.8686 & 0.8531 & 0.8380 & 0.8231 & 0.8085 & \\
\hline & 0.2 & 1.2089 & 1.1154 & 1.0291 & 0.9309 & 0.9124 & 0.8942 & 0.8764 & 0.8589 & 0.8417 & 0.8249 & 0.8085 & \\
\hline & 0.3 & 1.2089 & 1.1457 & 1.0858 & 1.0291 & 0.9283 & 0.9072 & 0.8865 & 0.8663 & 0.8466 & 0.8273 & 0.8085 & \\
\hline & 0.4 & 1.2089 & 1.1612 & 1.1154 & 1.0714 & 1.0291 & 0.9247 & 0.9002 & 0.8764 & 0.8531 & 0.8305 & 0.8085 & \\
\hline & 0.5 & 1.2089 & 1.1706 & 1.1335 & 1.0975 & 1.0628 & 1.0291 & 0.9198 & 0.8906 & 0.8624 & 0.8350 & 0.8085 & \\
\hline & 0.6 & 1.2089 & 1.1769 & 1.1457 & 1.1154 & 1.0858 & 1.0571 & 1.0291 & 0.9124 & 0.8764 & 0.8417 & 0.8085 & \\
\hline & 0.7 & 1.2089 & 1.1814 & 1.1545 & 1.1283 & 1.1026 & 1.0775 & 1.0530 & 1.0291 & 0.9002 & 0.8531 & 0.8085 & \\
\hline & 0.8 & 1.2089 & 1.1848 & 1.1612 & 1.1380 & 1.1154 & 1.0931 & 1.0714 & 1.0500 & 1.0291 & 0.8764 & 0.8085 & \\
\hline & 0.9 & 1.2089 & 1.1875 & 1.1664 & 1.1457 & 1.1254 & 1.1054 & 1.0858 & 1.0666 & 1.0477 & 1.0291 & 0.8085 & \\
\hline & 1 & 1.2089 & 1.1896 & 1.1706 & 1.1519 & 1.1335 & 1.1154 & 1.0975 & 1.0800 & 1.0628 & 1.0458 & 1.0291 & \\
\hline & 0 & 0.6748 & 0.6636 & 0.6526 & 0.6417 & 0.6310 & 0.6205 & 0.6102 & 0.6000 & 0.5900 & 0.5802 & 0.5705 & \multirow{3}{*}{ 恿 } \\
\hline & 0.1 & 0.8676 & 0.7337 & 0.6624 & 0.6501 & 0.6381 & 0.6263 & 0.6148 & 0.6034 & 0.5922 & 0.5813 & 0.5705 & \\
\hline & 0.2 & 0.8676 & 0.7978 & 0.7337 & 0.6608 & 0.6471 & 0.6337 & 0.6205 & 0.6076 & 0.5950 & 0.5826 & 0.5705 & \\
\hline
\end{tabular}




\begin{tabular}{|c|c|c|c|c|c|c|c|c|c|c|c|c|}
\hline 0.3 & 0.8676 & 0.8204 & 0.7759 & 0.7337 & 0.6588 & 0.6432 & 0.6280 & 0.6131 & 0.5986 & 0.5844 & 0.5705 & \\
\hline 0.4 & 0.8676 & 0.8320 & 0.7978 & 0.7651 & 0.7337 & 0.6562 & 0.6381 & 0.6205 & 0.6034 & 0.5867 & 0.5705 & \\
\hline 0.5 & 0.8676 & 0.8390 & 0.8113 & 0.7846 & 0.7587 & 0.7337 & 0.6526 & 0.6310 & 0.6102 & 0.5900 & 0.5705 & \\
\hline 0.6 & 0.8676 & 0.8437 & 0.8204 & 0.7978 & 0.7759 & 0.7545 & 0.7337 & 0.6471 & 0.6205 & 0.5950 & 0.5705 & \\
\hline 0.7 & 0.8676 & 0.8470 & 0.8270 & 0.8074 & 0.7883 & 0.7697 & 0.7515 & 0.7337 & 0.6381 & 0.6034 & 0.5705 & \\
\hline 0.8 & 0.8676 & 0.8496 & 0.8320 & 0.8147 & 0.7978 & 0.7813 & 0.7651 & 0.7493 & 0.7337 & 0.6205 & 0.5705 & \\
\hline 0.9 & 0.8676 & 0.8516 & 0.8358 & 0.8204 & 0.8053 & 0.7904 & 0.7759 & 0.7616 & 0.7475 & 0.7337 & 0.5705 & \\
\hline 1 & 0.8676 & 0.8531 & 0.8390 & 0.8250 & 0.8113 & 0.7978 & 0.7846 & 0.7715 & 0.7587 & 0.7461 & 0.7337 & \\
\hline 0 & 0.9609 & 0.9456 & 0.9295 & 0.9135 & 0.8977 & 0.8823 & 0.8670 & 0.8521 & 0.8374 & 0.8229 & 0.8087 & \multirow{11}{*}{$\begin{array}{l}3 \\
\text { 采 } \\
8\end{array}$} \\
\hline 0.1 & 1.2214 & 1.0409 & 0.9439 & 0.9259 & 0.9082 & 0.8908 & 0.8738 & 0.8570 & 0.8406 & 0.8245 & 0.8087 & \\
\hline 0.2 & 1.2214 & 1.1274 & 1.0409 & 0.9417 & 0.9214 & 0.9016 & 0.8823 & 0.8633 & 0.8447 & 0.8265 & 0.8087 & \\
\hline 0.3 & 1.2214 & 1.1579 & 1.0977 & 1.0409 & 0.9388 & 0.9157 & 0.8933 & 0.8714 & 0.8500 & 0.8291 & 0.8087 & \\
\hline 0.4 & 1.2214 & 1.1735 & 1.1274 & 1.0832 & 1.0409 & 0.9349 & 0.9082 & 0.8823 & 0.8570 & 0.8325 & 0.8087 & \\
\hline 0.5 & 1.2214 & 1.1829 & 1.1456 & 1.1095 & 1.0746 & 1.0409 & 0.9295 & 0.8977 & 0.8670 & 0.8374 & 0.8087 & \\
\hline 0.6 & 1.2214 & 1.1892 & 1.1579 & 1.1274 & 1.0977 & 1.0689 & 1.0409 & 0.9214 & 0.8823 & 0.8447 & 0.8087 & \\
\hline 0.7 & 1.2214 & 1.1938 & 1.1668 & 1.1404 & 1.1146 & 1.0894 & 1.0648 & 1.0409 & 0.9082 & 0.8570 & 0.8087 & \\
\hline 0.8 & 1.2214 & 1.1972 & 1.1735 & 1.1502 & 1.1274 & 1.1051 & 1.0832 & 1.0618 & 1.0409 & 0.8823 & 0.8087 & \\
\hline 0.9 & 1.2214 & 1.1999 & 1.1787 & 1.1579 & 1.1375 & 1.1174 & 1.0977 & 1.0784 & 1.0594 & 1.0409 & 0.8087 & \\
\hline 1 & 1.2214 & 1.2020 & 1.1829 & 1.1641 & 1.1456 & 1.1274 & 1.1095 & 1.0919 & 1.0746 & 1.0576 & 1.0409 & \\
\hline 0 & 0.5263 & 0.5196 & 0.5130 & 0.5065 & 0.5000 & 0.4936 & 0.4873 & 0.4811 & 0.4749 & 0.4688 & 0.4628 & \multirow{11}{*}{ 防 } \\
\hline 0.1 & 0.6382 & 0.5619 & 0.5189 & 0.5116 & 0.5043 & 0.4972 & 0.4901 & 0.4831 & 0.4763 & 0.4695 & 0.4628 & \\
\hline 0.2 & 0.6382 & 0.5989 & 0.5619 & 0.5180 & 0.5097 & 0.5016 & 0.4936 & 0.4857 & 0.4780 & 0.4703 & 0.4628 & \\
\hline 0.3 & 0.6382 & 0.6117 & 0.5863 & 0.5619 & 0.5168 & 0.5074 & 0.4982 & 0.4891 & 0.4802 & 0.4714 & 0.4628 & \\
\hline 0.4 & 0.6382 & 0.6182 & 0.5989 & 0.5801 & 0.5619 & 0.5152 & 0.5043 & 0.4936 & 0.4831 & 0.4729 & 0.4628 & \\
\hline 0.5 & 0.6382 & 0.6222 & 0.6066 & 0.5913 & 0.5764 & 0.5619 & 0.5130 & 0.5000 & 0.4873 & 0.4749 & 0.4628 & \\
\hline 0.6 & 0.6382 & 0.6248 & 0.6117 & 0.5989 & 0.5863 & 0.5740 & 0.5619 & 0.5097 & 0.4936 & 0.4780 & 0.4628 & \\
\hline 0.7 & 0.6382 & 0.6267 & 0.6154 & 0.6044 & 0.5935 & 0.5828 & 0.5722 & 0.5619 & 0.5043 & 0.4831 & 0.4628 & \\
\hline 0.8 & 0.6382 & 0.6281 & 0.6182 & 0.6085 & 0.5989 & 0.5894 & 0.5801 & 0.5709 & 0.5619 & 0.4936 & 0.4628 & \\
\hline 0.9 & 0.6382 & 0.6293 & 0.6204 & 0.6117 & 0.6031 & 0.5947 & 0.5863 & 0.5781 & 0.5699 & 0.5619 & 0.4628 & \\
\hline 1 & 0.6382 & 0.6301 & 0.6222 & 0.6143 & 0.6066 & 0.5989 & 0.5913 & 0.5838 & 0.5764 & 0.5691 & 0.5619 & \\
\hline
\end{tabular}

Table 4. The First Stage Efficiency Scores of MFs Based on AFNDEA Approach

\begin{tabular}{|c|c|c|c|c|c|c|c|c|c|c|c|c|c|}
\hline \multirow{15}{*}{ 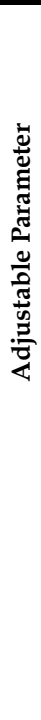 } & & \multicolumn{11}{|c|}{ Confidence Level } & \multirow{13}{*}{$\begin{array}{l}3 \\
\text { 是 }\end{array}$} \\
\hline & & 0 & 0.1 & 0.2 & 0.3 & 0.4 & 0.5 & 0.6 & 0.7 & 0.8 & 0.9 & 1 & \\
\hline & 0 & 0.9609 & 0.9456 & 0.9306 & 0.9158 & 0.9012 & 0.8868 & 0.8727 & 0.8588 & 0.8451 & 0.8317 & 0.8184 & \\
\hline & 0.1 & 1.2219 & 1.0407 & 0.9439 & 0.9273 & 0.9109 & 0.8948 & 0.8790 & 0.8634 & 0.8482 & 0.8332 & 0.8184 & \\
\hline & 0.2 & 1.2219 & 1.1276 & 1.0407 & 0.9418 & 0.9231 & 0.9048 & 0.8868 & 0.8692 & 0.8520 & 0.8350 & 0.8184 & \\
\hline & 0.3 & 1.2219 & 1.1582 & 1.0978 & 1.0407 & 0.9392 & 0.9179 & 0.8971 & 0.8767 & 0.8569 & 0.8374 & 0.8184 & \\
\hline & 0.4 & 1.2219 & 1.1738 & 1.1276 & 1.0833 & 1.0407 & 0.9356 & 0.9109 & 0.8868 & 0.8634 & 0.8406 & 0.8184 & \\
\hline & 0.5 & 1.2219 & 1.1832 & 1.1458 & 1.1096 & 1.0746 & 1.0407 & 0.9306 & 0.9012 & 0.8727 & 0.8451 & 0.8184 & \\
\hline & 0.6 & 1.2219 & 1.1896 & 1.1582 & 1.1276 & 1.0978 & 1.0689 & 1.0407 & 0.9231 & 0.8868 & 0.8520 & 0.8184 & \\
\hline & 0.7 & 1.2219 & 1.1942 & 1.1671 & 1.1406 & 1.1147 & 1.0895 & 1.0648 & 1.0407 & 0.9109 & 0.8634 & 0.8184 & \\
\hline & 0.8 & 1.2219 & 1.1976 & 1.1738 & 1.1505 & 1.1276 & 1.1052 & 1.0833 & 1.0618 & 1.0407 & 0.8868 & 0.8184 & \\
\hline & 0.9 & 1.2219 & 1.2003 & 1.1790 & 1.1582 & 1.1377 & 1.1176 & 1.0978 & 1.0784 & 1.0594 & 1.0407 & 0.8184 & \\
\hline & 1 & 1.2219 & 1.2024 & 1.1832 & 1.1644 & 1.1458 & 1.1276 & 1.1096 & 1.0920 & 1.0746 & 1.0575 & 1.0407 & \\
\hline & 0 & 0.6018 & 0.5922 & 0.5828 & 0.5735 & 0.5644 & 0.5554 & 0.5466 & 0.5379 & 0.5293 & 0.5209 & 0.5126 & \\
\hline & 0.1 & 0.7656 & 0.6521 & 0.5912 & 0.5807 & 0.5705 & 0.5604 & 0.5505 & 0.5408 & 0.5312 & 0.5218 & 0.5126 & \\
\hline
\end{tabular}




\begin{tabular}{|c|c|c|c|c|c|c|c|c|c|c|c|c|}
\hline 0.2 & 0.7656 & 0.7066 & 0.6521 & 0.5899 & 0.5782 & 0.5667 & 0.5554 & 0.5444 & 0.5336 & 0.5230 & 0.5126 & \\
\hline 0.3 & 0.7656 & 0.7257 & 0.6879 & 0.6521 & 0.5882 & 0.5749 & 0.5618 & 0.5491 & 0.5367 & 0.5245 & 0.5126 & \\
\hline 0.4 & 0.7656 & 0.7355 & 0.7066 & 0.6788 & 0.6521 & 0.5859 & 0.5705 & 0.5554 & 0.5408 & 0.5265 & 0.5126 & \\
\hline 0.5 & 0.7656 & 0.7414 & 0.7180 & 0.6953 & 0.6734 & 0.6521 & 0.5828 & 0.5644 & 0.5466 & 0.5293 & 0.5126 & \\
\hline 0.6 & 0.7656 & 0.7454 & 0.7257 & 0.7066 & 0.6879 & 0.6698 & 0.6521 & 0.5782 & 0.5554 & 0.5336 & 0.5126 & \\
\hline 0.7 & 0.7656 & 0.7482 & 0.7313 & 0.7147 & 0.6985 & 0.6827 & 0.6672 & 0.6521 & 0.5705 & 0.5408 & 0.5126 & \\
\hline 0.8 & 0.7656 & 0.7504 & 0.7355 & 0.7209 & 0.7066 & 0.6925 & 0.6788 & 0.6653 & 0.6521 & 0.5554 & 0.5126 & \\
\hline 0.9 & 0.7656 & 0.7520 & 0.7388 & 0.7257 & 0.7129 & 0.7003 & 0.6879 & 0.6758 & 0.6639 & 0.6521 & 0.5126 & \\
\hline 1 & 0.7656 & 0.7534 & 0.7414 & 0.7296 & 0.7180 & 0.7066 & 0.6953 & 0.6843 & 0.6734 & 0.6627 & 0.6521 & \\
\hline 0 & 0.4275 & 0.4206 & 0.4138 & 0.4071 & 0.4004 & 0.3939 & 0.3875 & 0.3812 & 0.3750 & 0.3689 & 0.3629 & \multirow{11}{*}{ 恿 } \\
\hline 0.1 & 0.5466 & 0.4641 & 0.4198 & 0.4123 & 0.4048 & 0.3975 & 0.3904 & 0.3833 & 0.3764 & 0.3696 & 0.3629 & \\
\hline 0.2 & 0.5466 & 0.5036 & 0.4641 & 0.4189 & 0.4104 & 0.4021 & 0.3939 & 0.3860 & 0.3781 & 0.3705 & 0.3629 & \\
\hline 0.3 & 0.5466 & 0.5176 & 0.4901 & 0.4641 & 0.4177 & 0.4080 & 0.3986 & 0.3894 & 0.3803 & 0.3715 & 0.3629 & \\
\hline 0.4 & 0.5466 & 0.5247 & 0.5036 & 0.4834 & 0.4641 & 0.4160 & 0.4048 & 0.3939 & 0.3833 & 0.3730 & 0.3629 & \\
\hline 0.5 & 0.5466 & 0.5290 & 0.5119 & 0.4955 & 0.4795 & 0.4641 & 0.4138 & 0.4004 & 0.3875 & 0.3750 & 0.3629 & \\
\hline 0.6 & 0.5466 & 0.5319 & 0.5176 & 0.5036 & 0.4901 & 0.4769 & 0.4641 & 0.4104 & 0.3939 & 0.3781 & 0.3629 & \\
\hline 0.7 & 0.5466 & 0.5340 & 0.5216 & 0.5096 & 0.4978 & 0.4863 & 0.4750 & 0.4641 & 0.4048 & 0.3833 & 0.3629 & \\
\hline 0.8 & 0.5466 & 0.5355 & 0.5247 & 0.5140 & 0.5036 & 0.4934 & 0.4834 & 0.4736 & 0.4641 & 0.3939 & 0.3629 & \\
\hline 0.9 & 0.5466 & 0.5368 & 0.5271 & 0.5176 & 0.5082 & 0.4991 & 0.4901 & 0.4812 & 0.4726 & 0.4641 & 0.3629 & \\
\hline 1 & 0.5466 & 0.5377 & 0.5290 & 0.5204 & 0.5119 & 0.5036 & 0.4955 & 0.4874 & 0.4795 & 0.4717 & 0.4641 & \\
\hline 0 & 0.9606 & 0.9455 & 0.9307 & 0.9166 & 0.9028 & 0.8891 & 0.8757 & 0.8625 & 0.8494 & 0.8366 & 0.8239 & \multirow{11}{*}{3} \\
\hline 0.1 & 1.2206 & 1.0423 & 0.9438 & 0.9275 & 0.9120 & 0.8967 & 0.8817 & 0.8669 & 0.8523 & 0.8380 & 0.8239 & \\
\hline 0.2 & 1.2206 & 1.1272 & 1.0423 & 0.9417 & 0.9236 & 0.9062 & 0.8891 & 0.8724 & 0.8559 & 0.8398 & 0.8239 & \\
\hline 0.3 & 1.2206 & 1.1575 & 1.0977 & 1.0423 & 0.9390 & 0.9186 & 0.8989 & 0.8795 & 0.8606 & 0.8421 & 0.8239 & \\
\hline 0.4 & 1.2206 & 1.1729 & 1.1272 & 1.0832 & 1.0423 & 0.9355 & 0.9120 & 0.8891 & 0.8669 & 0.8451 & 0.8239 & \\
\hline 0.5 & 1.2206 & 1.1823 & 1.1452 & 1.1094 & 1.0746 & 1.0423 & 0.9307 & 0.9028 & 0.8757 & 0.8494 & 0.8239 & \\
\hline 0.6 & 1.2206 & 1.1886 & 1.1575 & 1.1272 & 1.0977 & 1.0691 & 1.0423 & 0.9236 & 0.8891 & 0.8559 & 0.8239 & \\
\hline 0.7 & 1.2206 & 1.1931 & 1.1663 & 1.1401 & 1.1144 & 1.0894 & 1.0652 & 1.0423 & 0.9120 & 0.8669 & 0.8239 & \\
\hline 0.8 & 1.2206 & 1.1965 & 1.1729 & 1.1498 & 1.1272 & 1.1050 & 1.0832 & 1.0623 & 1.0423 & 0.8891 & 0.8239 & \\
\hline 0.9 & 1.2206 & 1.1992 & 1.1781 & 1.1575 & 1.1372 & 1.1172 & 1.0977 & 1.0784 & 1.0601 & 1.0423 & 0.8239 & \\
\hline 1 & 1.2206 & 1.2013 & 1.1823 & 1.1636 & 1.1452 & 1.1272 & 1.1094 & 1.0919 & 1.0746 & 1.0583 & 1.0423 & \\
\hline 0 & 0.9606 & 0.9453 & 0.9303 & 0.9156 & 0.9010 & 0.8867 & 0.8726 & 0.8587 & 0.8451 & 0.8316 & 0.8184 & \multirow{11}{*}{ 鼻 } \\
\hline 0.1 & 1.2262 & 1.0440 & 0.9437 & 0.9270 & 0.9107 & 0.8946 & 0.8788 & 0.8633 & 0.8481 & 0.8331 & 0.8184 & \\
\hline 0.2 & 1.2262 & 1.1314 & 1.0440 & 0.9416 & 0.9229 & 0.9046 & 0.8867 & 0.8691 & 0.8519 & 0.8350 & 0.8184 & \\
\hline 0.3 & 1.2262 & 1.1621 & 1.1015 & 1.0440 & 0.9389 & 0.9176 & 0.8969 & 0.8766 & 0.8568 & 0.8373 & 0.8184 & \\
\hline 0.4 & 1.2262 & 1.1778 & 1.1314 & 1.0868 & 1.0440 & 0.9353 & 0.9107 & 0.8867 & 0.8633 & 0.8406 & 0.8184 & \\
\hline 0.5 & 1.2262 & 1.1873 & 1.1497 & 1.1133 & 1.0781 & 1.0440 & 0.9303 & 0.9010 & 0.8726 & 0.8451 & 0.8184 & \\
\hline 0.6 & 1.2262 & 1.1937 & 1.1621 & 1.1314 & 1.1015 & 1.0723 & 1.0440 & 0.9229 & 0.8867 & 0.8519 & 0.8184 & \\
\hline 0.7 & 1.2262 & 1.1983 & 1.1711 & 1.1445 & 1.1185 & 1.0931 & 1.0682 & 1.0440 & 0.9107 & 0.8633 & 0.8184 & \\
\hline 0.8 & 1.2262 & 1.2018 & 1.1778 & 1.1544 & 1.1314 & 1.1089 & 1.0868 & 1.0652 & 1.0440 & 0.8867 & 0.8184 & \\
\hline 0.9 & 1.2262 & 1.2045 & 1.1831 & 1.1621 & 1.1415 & 1.1213 & 1.1015 & 1.0820 & 1.0628 & 1.0440 & 0.8184 & \\
\hline 1 & 1.2262 & 1.2066 & 1.1873 & 1.1684 & 1.1497 & 1.1314 & 1.1133 & 1.0956 & 1.0781 & 1.0609 & 1.0440 & \\
\hline
\end{tabular}

Table 5. The Second Stage Efficiency Scores of MFs Based on AFNDEA Approach

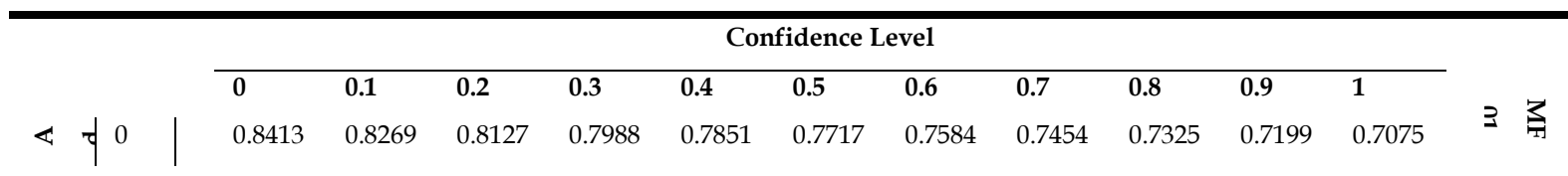




\begin{tabular}{|c|c|c|c|c|c|c|c|c|c|c|c|c|}
\hline 0.1 & 1.0887 & 0.9172 & 0.8253 & 0.8096 & 0.7942 & 0.7791 & 0.7643 & 0.7497 & 0.7354 & 0.7213 & 0.7075 & \\
\hline 0.2 & 1.0887 & 0.9994 & 0.9172 & 0.8233 & 0.8057 & 0.7885 & 0.7717 & 0.7551 & 0.7389 & 0.7230 & 0.7075 & \\
\hline 0.3 & 1.0887 & 1.0283 & 0.9712 & 0.9172 & 0.8208 & 0.8008 & 0.7813 & 0.7622 & 0.7435 & 0.7253 & 0.7075 & \\
\hline 0.4 & 1.0887 & 1.0431 & 0.9994 & 0.9574 & 0.9172 & 0.8174 & 0.7942 & 0.7717 & 0.7497 & 0.7283 & 0.7075 & \\
\hline 0.5 & 1.0887 & 1.0521 & 1.0166 & 0.9824 & 0.9493 & 0.9172 & 0.8127 & 0.7851 & 0.7584 & 0.7325 & 0.7075 & \\
\hline 0.6 & 1.0887 & 1.0581 & 1.0283 & 0.9994 & 0.9712 & 0.9439 & 0.9172 & 0.8057 & 0.7717 & 0.7389 & 0.7075 & \\
\hline 0.7 & 1.0887 & 1.0624 & 1.0367 & 1.0117 & 0.9872 & 0.9633 & 0.9400 & 0.9172 & 0.7942 & 0.7497 & 0.7075 & \\
\hline 0.8 & 1.0887 & 1.0656 & 1.0431 & 1.0210 & 0.9994 & 0.9782 & 0.9574 & 0.9371 & 0.9172 & 0.7717 & 0.7075 & \\
\hline 0.9 & 1.0887 & 1.0682 & 1.0481 & 1.0283 & 1.0089 & 0.9899 & 0.9712 & 0.9529 & 0.9349 & 0.9172 & 0.7075 & \\
\hline 1 & 1.0887 & 1.0702 & 1.0521 & 1.0342 & 1.0166 & 0.9994 & 0.9824 & 0.9657 & 0.9493 & 0.9331 & 0.9172 & \\
\hline 0 & 0.9578 & 0.9426 & 0.9276 & 0.9129 & 0.8984 & 0.8841 & 0.8700 & 0.8562 & 0.8426 & 0.8291 & 0.8159 & \multirow{11}{*}{$\begin{array}{l}3 \\
\text { 구 } \\
\text { ำ }\end{array}$} \\
\hline 0.1 & 1.2176 & 1.0373 & 0.9409 & 0.9243 & 0.9080 & 0.8920 & 0.8762 & 0.8608 & 0.8456 & 0.8306 & 0.8159 & \\
\hline 0.2 & 1.2176 & 1.1238 & 1.0373 & 0.9388 & 0.9202 & 0.9020 & 0.8841 & 0.8665 & 0.8493 & 0.8325 & 0.8159 & \\
\hline 0.3 & 1.2176 & 1.1542 & 1.0942 & 1.0373 & 0.9362 & 0.9150 & 0.8943 & 0.8740 & 0.8542 & 0.8349 & 0.8159 & \\
\hline 0.4 & 1.2176 & 1.1697 & 1.1238 & 1.0796 & 1.0373 & 0.9326 & 0.9080 & 0.8841 & 0.8608 & 0.8381 & 0.8159 & \\
\hline 0.5 & 1.2176 & 1.1792 & 1.1419 & 1.1059 & 1.0710 & 1.0373 & 0.9276 & 0.8984 & 0.8700 & 0.8426 & 0.8159 & \\
\hline 0.6 & 1.2176 & 1.1855 & 1.1542 & 1.1238 & 1.0942 & 1.0653 & 1.0373 & 0.9202 & 0.8841 & 0.8493 & 0.8159 & \\
\hline 0.7 & 1.2176 & 1.1900 & 1.1631 & 1.1367 & 1.1110 & 1.0858 & 1.0613 & 1.0373 & 0.9080 & 0.8608 & 0.8159 & \\
\hline 0.8 & 1.2176 & 1.1934 & 1.1697 & 1.1465 & 1.1238 & 1.1015 & 1.0796 & 1.0582 & 1.0373 & 0.8841 & 0.8159 & \\
\hline 0.9 & 1.2176 & 1.1961 & 1.1750 & 1.1542 & 1.1338 & 1.1138 & 1.0942 & 1.0749 & 1.0559 & 1.0373 & 0.8159 & \\
\hline 1 & 1.2176 & 1.1982 & 1.1792 & 1.1604 & 1.1419 & 1.1238 & 1.1059 & 1.0883 & 1.0710 & 1.0540 & 1.0373 & \\
\hline 0 & 0.7372 & 0.7253 & 0.7137 & 0.7022 & 0.6909 & 0.6798 & 0.6688 & 0.6581 & 0.6475 & 0.6370 & 0.6268 & \multirow{11}{*}{3} \\
\hline 0.1 & 0.9402 & 0.7993 & 0.7240 & 0.7111 & 0.6984 & 0.6859 & 0.6737 & 0.6616 & 0.6498 & 0.6382 & 0.6268 & \\
\hline 0.2 & 0.9402 & 0.8668 & 0.7993 & 0.7224 & 0.7079 & 0.6937 & 0.6798 & 0.6661 & 0.6527 & 0.6396 & 0.6268 & \\
\hline 0.3 & 0.9402 & 0.8906 & 0.8437 & 0.7993 & 0.7203 & 0.7038 & 0.6877 & 0.6719 & 0.6565 & 0.6415 & 0.6268 & \\
\hline 0.4 & 0.9402 & 0.9028 & 0.8668 & 0.8324 & 0.7993 & 0.7175 & 0.6984 & 0.6798 & 0.6616 & 0.6440 & 0.6268 & \\
\hline 0.5 & 0.9402 & 0.9101 & 0.8810 & 0.8529 & 0.8256 & 0.7993 & 0.7137 & 0.6909 & 0.6688 & 0.6475 & 0.6268 & \\
\hline 0.6 & 0.9402 & 0.9151 & 0.8906 & 0.8668 & 0.8437 & 0.8212 & 0.7993 & 0.7079 & 0.6798 & 0.6527 & 0.6268 & \\
\hline 0.7 & 0.9402 & 0.9186 & 0.8975 & 0.8769 & 0.8568 & 0.8372 & 0.8180 & 0.7993 & 0.6984 & 0.6616 & 0.6268 & \\
\hline 0.8 & 0.9402 & 0.9213 & 0.9028 & 0.8846 & 0.8668 & 0.8494 & 0.8324 & 0.8156 & 0.7993 & 0.6798 & 0.6268 & \\
\hline 0.9 & 0.9402 & 0.9234 & 0.9068 & 0.8906 & 0.8747 & 0.8590 & 0.8437 & 0.8286 & 0.8138 & 0.7993 & 0.6268 & \\
\hline 1 & 0.9402 & 0.9250 & 0.9101 & 0.8955 & 0.8810 & 0.8668 & 0.8529 & 0.8391 & 0.8256 & 0.8123 & 0.7993 & \\
\hline 0 & 0.9609 & 0.9457 & 0.9292 & 0.9128 & 0.8967 & 0.8808 & 0.8652 & 0.8498 & 0.8347 & 0.8198 & 0.8052 & \multirow{11}{*}{ 主 } \\
\hline 0.1 & 1.2216 & 1.0406 & 0.9440 & 0.9256 & 0.9074 & 0.8896 & 0.8721 & 0.8549 & 0.8380 & 0.8215 & 0.8052 & \\
\hline 0.2 & 1.2216 & 1.1275 & 1.0406 & 0.9417 & 0.9210 & 0.9007 & 0.8808 & 0.8613 & 0.8422 & 0.8235 & 0.8052 & \\
\hline 0.3 & 1.2216 & 1.1580 & 1.0977 & 1.0406 & 0.9387 & 0.9152 & 0.8921 & 0.8696 & 0.8476 & 0.8262 & 0.8052 & \\
\hline 0.4 & 1.2216 & 1.1736 & 1.1275 & 1.0832 & 1.0406 & 0.9348 & 0.9074 & 0.8808 & 0.8549 & 0.8297 & 0.8052 & \\
\hline 0.5 & 1.2216 & 1.1830 & 1.1457 & 1.1095 & 1.0745 & 1.0406 & 0.9292 & 0.8967 & 0.8652 & 0.8347 & 0.8052 & \\
\hline 0.6 & 1.2216 & 1.1893 & 1.1580 & 1.1275 & 1.0977 & 1.0688 & 1.0406 & 0.9210 & 0.8808 & 0.8422 & 0.8052 & \\
\hline 0.7 & 1.2216 & 1.1939 & 1.1669 & 1.1404 & 1.1146 & 1.0894 & 1.0647 & 1.0406 & 0.9074 & 0.8549 & 0.8052 & \\
\hline 0.8 & 1.2216 & 1.1973 & 1.1736 & 1.1503 & 1.1275 & 1.1051 & 1.0832 & 1.0617 & 1.0406 & 0.8808 & 0.8052 & \\
\hline 0.9 & 1.2216 & 1.2000 & 1.1788 & 1.1580 & 1.1375 & 1.1175 & 1.0977 & 1.0784 & 1.0593 & 1.0406 & 0.8052 & \\
\hline 1 & 1.2216 & 1.2021 & 1.1830 & 1.1642 & 1.1457 & 1.1275 & 1.1095 & 1.0919 & 1.0745 & 1.0574 & 1.0406 & \\
\hline 0 & 0.0938 & 0.0923 & 0.0908 & 0.0894 & 0.0880 & 0.0866 & 0.0852 & 0.0838 & 0.0825 & 0.0811 & 0.0798 & \multirow{6}{*}{$\begin{array}{l}\text { 光 } \\
\text { \& }\end{array}$} \\
\hline 0.1 & 0.1195 & 0.1017 & 0.0922 & 0.0905 & 0.0889 & 0.0873 & 0.0858 & 0.0843 & 0.0828 & 0.0813 & 0.0798 & \\
\hline 0.2 & 0.1195 & 0.1102 & 0.1017 & 0.0919 & 0.0901 & 0.0883 & 0.0866 & 0.0848 & 0.0831 & 0.0815 & 0.0798 & \\
\hline 0.3 & 0.1195 & 0.1132 & 0.1073 & 0.1017 & 0.0917 & 0.0896 & 0.0876 & 0.0856 & 0.0836 & 0.0817 & 0.0798 & \\
\hline 0.4 & 0.1195 & 0.1147 & 0.1102 & 0.1059 & 0.1017 & 0.0913 & 0.0889 & 0.0866 & 0.0843 & 0.0820 & 0.0798 & \\
\hline 0.5 & 0.1195 & 0.1157 & 0.1120 & 0.1085 & 0.1050 & 0.1017 & 0.0908 & 0.0880 & 0.0852 & 0.0825 & 0.0798 & \\
\hline
\end{tabular}




\begin{tabular}{|l|lllllllllll}
0.6 & 0.1195 & 0.1163 & 0.1132 & 0.1102 & 0.1073 & 0.1044 & 0.1017 & 0.0901 & 0.0866 & 0.0831 & 0.0798 \\
0.7 & 0.1195 & 0.1167 & 0.1141 & 0.1115 & 0.1090 & 0.1065 & 0.1041 & 0.1017 & 0.0889 & 0.0843 & 0.0798 \\
0.8 & 0.1195 & 0.1171 & 0.1147 & 0.1125 & 0.1102 & 0.1080 & 0.1059 & 0.1038 & 0.1017 & 0.0866 & 0.0798 \\
0.9 & 0.1195 & 0.1173 & 0.1153 & 0.1132 & 0.1112 & 0.1092 & 0.1073 & 0.1054 & 0.1035 & 0.1017 & 0.0798 \\
1 & 0.1195 & 0.1176 & 0.1157 & 0.1138 & 0.1120 & 0.1102 & 0.1085 & 0.1067 & 0.1050 & 0.1033 & 0.1017
\end{tabular}

As it can be seen from Tables 3 to 5, by increasing the optimistic-pessimistic parameter from 0 to 1 , the attitudes of decision maker can be changed from pessimistic to optimistic viewpoint. Also, for the same optimistic-pessimistic parameter $(\beta)$, the efficiency scores of DMUs and sub-DMUs decrease while the confidence level $(\alpha)$ increase. Moreover, as expected, minimum efficiency score (worst case) for each DMUs and sub-DMUs occurred for pair of $(\alpha, \beta)=(1,0)$, and maximum efficiency score (best case) for each DMUs and sub-DMUs occurred for pair of $(\alpha, \beta)=(0,1)$.

After calculation of $\Psi_{p(\alpha, \beta)}^{\text {Overall* }^{*}}, \Psi_{p(\alpha, \beta)}^{\text {Stage } 1^{*}}$, and $\Psi_{p(\alpha, \beta)}^{\text {Stage } 2^{*}}$ using Model (18), Model (19), and Equation (20), respectively, all the MFs from overall, operational, and portfolio functions can be fully ranked. It should be noted that the graphical presentation of efficiency surface for all MFs are available in Supplementary Material. The ranking of mutual funds based on proposed ranking method is presented in Table 6 .

Table 6. The Ranking of MFs under Fuzzy Environment

\begin{tabular}{ccccccc}
\hline \multirow{2}{*}{ MFs } & \multicolumn{2}{c}{ Overall } & \multicolumn{2}{c}{ First Stage } & \multicolumn{2}{c}{ Second Stage } \\
\cline { 2 - 7 } & Criterion & Rank & Criterion & Rank & Criterion & Rank \\
\hline MF 01 & 0.9544 & 3 & 1.0123 & 3 & 0.8904 & 3 \\
MF 02 & 1.0009 & 2 & 0.6342 & 4 & 1.0090 & 1 \\
MF 03 & 0.7130 & 4 & 0.4511 & 5 & 0.7773 & 4 \\
MF 04 & 1.0097 & 1 & 1.0135 & 2 & 1.0089 & 2 \\
MF 05 & 0.5483 & 5 & 1.0143 & 1 & 0.0989 & 5 \\
\hline
\end{tabular}

According to results obtained from Table 6, MF 04 has the best overall performance in comparison with other mutual funds. Also, MF 05 and MF 02 have the best performance in operational management and portfolio management, respectively. It is important to note that lower costs do not always lead DMU to an efficient performance. In other words, by proper costing, the performance of system can be significantly improved. For example, MF 04 despite the highest cost in the 
management department, has been able to achieve acceptable stability and performance due to the employ of experienced managers and this point can be considered as a benchmark for other MFs.

Finally, the results of proposed FNDEA approach in this study are compared to the results of NDEA approach under crisp (certain) data. Accordingly, for comparing the proposed FNDEA and ranking method with the traditional NDEA, firstly, all fuzzy data should be converted to the crisp numbers.

It should be noted that for defuzzification of all trapezoidal fuzzy numbers that presented in Table 2, the expected value (EV) approach is utilized (Heilpern, 1992). The crisp data set for MFs are shown in Table 7.

Table 7. Crisp Data Set for 5 Mutual Funds in Iran

\begin{tabular}{cccccc}
\hline Index & MF 01 & MF 02 & MF 03 & MF 04 & MF 05 \\
\hline X1 & 0.1865 & 1.5562 & 0.8428 & 4.4909 & 0.2373 \\
X2 & 174.9668 & 5.3561 & 82.9641 & 0.0139 & 0.0123 \\
\hline Z & 2.6362 & 10.3859 & 4.3379 & 16.1692 & 2.5147 \\
\hline G1 & 0.0122 & 0.1404 & 0.0904 & 0.1842 & 0.0127 \\
G2 & 3.4000 & 4.9000 & 4.4300 & 4.5000 & 9.2000 \\
G3 & 0.6245 & 0.3449 & 0.2876 & 0.4341 & 0.1912 \\
G4 & 1.2200 & 0.9800 & 2.5500 & 2.1200 & 2.5000 \\
\hline Y & 58.4400 & 39.9800 & 24.7600 & 52.0000 & 5.5200 \\
\hline
\end{tabular}

Now, the traditional NDEA approach is implemented for crisp data. The results and ranking of MFs under certainty obtained from Models (8) and (10) as well as Equation (11) are introduced in Table 8.

Table 8. The Ranking of MFs under Crisp Data

\begin{tabular}{ccccccc}
\hline \multirow{2}{*}{ MFs } & \multicolumn{2}{c}{ Overall } & \multicolumn{2}{c}{ First Stage } & \multicolumn{2}{c}{ Second Stage } \\
\cline { 2 - 7 } & Efficiency & Rank & Efficiency & Rank & Efficiency & Rank \\
\hline MF 01 & 0.9423 & 3 & 1.0000 & 1 & 0.8782 & 3 \\
MF 02 & 0.9886 & 2 & 0.6265 & 4 & 0.9967 & 2 \\
MF 03 & 0.7037 & 4 & 0.4454 & 5 & 0.7676 & 4 \\
MF 04 & 1.0000 & 1 & 1.0000 & 1 & 1.0000 & 1 \\
MF 05 & 0.5435 & 5 & 1.0000 & 1 & 0.0977 & 5 \\
\hline
\end{tabular}


From Tables 6 and 8, it can be clearly observed that the overall ranking based on both FNDEA and NDEA methods are same and this point indicate on the validation and verification of proposed FNDEA approach.

Also, discriminatory power of proposed FNDEA and ranking method is more than traditional NDEA approach. In other words, the proposed ranking method based on AFNDEA approach is capable to fully rank all the mutual funds from overall, stage 1, and stage 2 viewpoints, while classic NDEA approach does not have the ability to rank efficient DMUs. Moreover, the FNDEA approach is flexible, applicable, general and adjustable for different DMs.

\section{Discussion and Conclusions}

In this study, a new fuzzy network DEA approach and novel ranking method using an adjustable possibility approach and chance-constrained programming was proposed. This approach is presented for extended two-stage structure contain of leakage variables and added inputs to the second stage. For solving and showing validation of the proposed adjustable possibilistic network DEA approach and ranking method, a real case study of mutual funds in Iranian financial market was applied.

Experimental results show that the proposed AFNDEA model and ranking method are effective and applicable for assessment and ranking of all MFs under data ambiguity. It should be noted that presented adjustable fuzzy NDEA model as well as ranking method can be implemented by mangers for each real-world application such as supply chain, insurance, banking, transportation, manufacturing, education, energy, environmental, health care, information technology, power, etc. that data are fuzzy or linguistic variables (for more details see FNDEA studies that presented in Table 1).

The main advantages of proposed AFNDEA and ranking method can be summarized as follows: considering all preferences of DM from pessimistic to optimistic attitude only by setting an adjustable parameter, capability to full rank of all two-stage DMUs from overall and stages viewpoints, linearity of proposed 
AFNDEA approach, unique efficiency decomposing under fuzzy environment, capability to extending for other network structures such as series and parallel. Remarkably, in this study, the FNDEA approach is proposed based on adjustable possibilistic programming for the first time.

For the future research directions, the presented AFNDEA approach and ranking method could be extended for other network structures such as series, parallel, and mixed (for more details see Cook et al., 2010b; Kao, 2009; 2012; 2014a; Despotis et al., 2016). Moreover, the uncertain network DEA models for dealing with uncertainty could be proposed based on other approaches of uncertain programming in literature such as robust optimization (for more details see Peykani et al., 2020).

\section{References}

Ameri, Z., Sana, S. S., \& Sheikh, R. (2019). Self-Assessment of Parallel Network Systems with Intuitionistic Fuzzy Data: A Case Study. Soft Computing, 23(23), 12821-12832.

Amirteimoori, A., Azizi, H., \& Kordrostami, S. (2020). Double frontier two-stage fuzzy data envelopment analysis. International Journal of Uncertainty, Fuzziness and Knowledge-Based Systems, 28(1), 117-152.

Banker, R. D., Charnes, A., \& Cooper, W. W. (1984). Some Models for Estimating Technical and Scale Inefficiencies in Data Envelopment Analysis. Management Science, 30(9), 1078-1092.

Castelli, L., Pesenti, R., \& Ukovich, W. (2010). A classification of DEA models when the internal structure of the decision making units is considered. Annals of Operations Research, 173(1), 207-235.

Charnes, A., \& Cooper, W. W. (1959). Chance-constrained programming. Management Science, 6(1), 73-79.

Charnes, A., \& Cooper, W. W. (1962). Programming with Linear Fractional Functionals. Naval Research Logistics Quarterly, 9(3-4), 181-186.

Charnes, A., Cooper, W. W., \& Rhodes, E. (1978). Measuring the Efficiency of Decision Making Units. European Journal of Operational Research, 2(6), 429-444.

Chen, Y., \& Zhu, J. (2004). Measuring Information Technology's Indirect Impact on Firm Performance. Information Technology and Management, 5(1-2), 9-22. 
Cook, W. D., \& Zhu, J. (2014). Data Envelopment Analysis: A Handbook of Modeling Internal Structure and Network. Springer.

Cook, W. D., Liang, L., \& Zhu, J. (2010a). Measuring performance of two-stage network structures by DEA: a review and future perspective. Omega, 38(6), 423430.

Cook, W. D., Zhu, J., Bi, G., \& Yang, F. (2010b). Network DEA: Additive efficiency decomposition. European Journal of Operational Research, 207(2), 1122-1129.

Emrouznejad, A., \& Amin, G. R. (2009). DEA models for ratio data: Convexity consideration. Applied Mathematical Modelling, 33(1), 486-498.

Emrouznejad, A., \& Tavana, M. (2014). Performance Measurement with Fuzzy Data Envelopment Analysis, Springer.

Emrouznejad, A., \& Yang, G. L. (2018). A survey and analysis of the first 40 years of scholarly literature in DEA: 1978-2016. Socio-Economic Planning Sciences, 61, 4-8.

Farrell, M. J. (1957). The Measurement of Productive Efficiency. Journal of the Royal Statistical Society: Series A (General), 120(3), 253-281.

Galagedera, D. U. (2019). Modelling Social Responsibility in Mutual Fund Performance Appraisal: A Two-Stage Data Envelopment Analysis Model with Non-Discretionary First Stage Output. European Journal of Operational Research, 273(1), 376-389.

Galagedera, D. U., Roshdi, I., Fukuyama, H., \& Zhu, J. (2018). A New Network DEA Model for Mutual Fund Performance Appraisal: An Application to US Equity Mutual Funds. Omega, 77, 168-179.

Galagedera, D. U., Watson, J., Premachandra, I. M., \& Chen, Y. (2016). Modeling Leakage in Two-Stage DEA Models: An Application to US Mutual Fund Families. Omega, 61, 62-77.

Halkos, G. E., Tzeremes, N. G., \& Kourtzidis, S. A. (2014). A unified classification of two-stage DEA models. Surveys in Operations Research and Management Science, $19(1), 1-16$.

Hanafizadeh, P., Khedmatgozar, H. R., Emrouznejad, A., \& Derakhshan, M. (2014). Neural network DEA for measuring the efficiency of mutual funds. International Journal of Applied Decision Sciences, 7(3), 255-269. 
Hatami-Marbini, A. (2019). Benchmarking with Network DEA in a Fuzzy Environment. RAIRO-Operations Research, 53(2), 687-703.

Hatami-Marbini, A., \& Saati, S. (2018). Efficiency Evaluation in Two-Stage Data Envelopment Analysis under a Fuzzy Environment: A Common-Weights Approach. Applied Soft Computing, 72, 156-165.

Hatami-Marbini, A., Emrouznejad, A., \& Tavana, M. (2011). A Taxonomy and Review of the Fuzzy Data Envelopment Analysis Literature: Two Decades in the Making. European Journal of Operational Research, 214(3), 457-472.

Hatami-Marbini, A., Saati, S., \& Sajadi, S. M. (2018). Efficiency Analysis in Two-Stage Structures using Fuzzy Data Envelopment Analysis. Central European Journal of Operations Research, 26, 909-932.

Heilpern, S. (1992). The expected value of a fuzzy number. Fuzzy Sets and Systems, $47(1), 81-86$.

Hemmati, M., Feiz, D., Jalilvand, M. R., \& Kholghi, I. (2016). Development of Fuzzy Two-Stage DEA Model for Competitive Advantage Based on RBV and Strategic Agility as a Dynamic Capability. Journal of Modelling in Management, 11(1), 288308.

Kao, C. (2009). Efficiency measurement for parallel production systems. European Journal of Operational Research, 196(3), 1107-1112.

Kao, C. (2012). Efficiency decomposition for parallel production systems. Journal of the Operational Research Society, 63(1), 64-71.

Despotis, D. K., Sotiros, D., \& Koronakos, G. (2016). A network DEA approach for series multi-stage processes. Omega, 61, 35-48.

Kao, C. (2014a). Network Data Envelopment Analysis: A Review. European Journal of Operational Research, 239(1), 1-16.

Kao, C. (2014b). Network Data Envelopment Analysis with Fuzzy Data. Performance Measurement with Fuzzy Data Envelopment Analysis, 191-206, Springer.

Kao, C. (2017). Network Data Envelopment Analysis. International Series in Operations Research \& Management, Springer.

Kao, C., \& Hwang, S. N. (2008). Efficiency Decomposition in Two-Stage Data Envelopment Analysis: An Application to Non-Life Insurance Companies in Taiwan. European Journal of Operational Research, 185(1), 418-429. 
Kao, C., \& Lin, P. H. (2012). Efficiency of Parallel Production Systems with Fuzzy Data. Fuzzy Sets and Systems, 198, 83-98.

Kao, C., \& Liu, S. T. (2011). Efficiencies of Two-Stage Systems with Fuzzy Data. Fuzzy Sets and Systems, 176(1), 20-35.

Khalili-Damghani, K., \& Taghavifard, M. (2012). A Three-Stage Fuzzy DEA Approach to Measure Performance of a Serial Process Including JIT Practices, Agility Indices, and Goals in Supply Chains. International Journal of Services and Operations Management, 13(2), 147-188.

Khalili-Damghani, K., \& Tavana, M. (2013). A New Fuzzy Network Data Envelopment Analysis Model for Measuring the Performance of Agility in Supply Chains. The International Journal of Advanced Manufacturing Technology, 69(1-4), 291-318.

Khalili-Damghani, K., Sadi-Nezhad, S., \& Hosseinzadeh Lotfi, F. (2014). Imprecise DEA Models to Assess the Agility of Supply Chains. Supply Chain Management under Fuzziness, 167-198, Springer, Berlin, Heidelberg.

Khalili-Damghani, K., Taghavifard, M., \& Abtahi, A. R. (2012). A Fuzzy Two-Stage DEA Approach for Performance Measurement: Real Case of Agility Performance in Dairy Supply Chains. International Journal of Applied Decision Sciences, 5(4), 293317.

Li, Y., Abtahi, A. R., \& Seyedan, M. (2019). Supply Chain Performance Evaluation Using Fuzzy Network Data Envelopment Analysis: A Case Study in Automotive Industry. Annals of Operations Research, 275(2), 461-484.

Liang, L., Cook, W. D., \& Zhu, J. (2008). DEA Models for Two-Stage Processes: Game Approach and Efficiency Decomposition. Naval Research Logistics, 55(7), 643-653.

Liu, S. T. (2014a). Fuzzy Efficiency Ranking in Fuzzy Two-Stage Data Envelopment Analysis. Optimization Letters, 8(2), 633-652.

Liu, S. T. (2014b). Restricting Weight Flexibility in Fuzzy Two-Stage DEA. Computers $\mathcal{E}$ Industrial Engineering, 74, 149-160.

Lotfi, F. H., Jahanshahloo, G. R., Vahidi, A. R., \& Dalirian, A. (2009). Efficiency and Effectiveness in Multi-Activity Network DEA Model with Fuzzy Data. Applied Mathematical Sciences, 3(52), 2603-2618.

Lozano, S. (2014). Process Efficiency of Two-Stage Systems with Fuzzy Data. Fuzzy Sets and Systems, 243, 36-49. 
Lozano, S., \& Moreno, P. (2013). A DEA Model for Two-Stage Systems with Fuzzy Data. IEEE International Conference on Fuzzy Systems, 1-6, IEEE.

Lozano, S., \& Moreno, P. (2014). Network Fuzzy Data Envelopment Analysis. Performance Measurement with Fuzzy Data Envelopment Analysis, 207-230, Springer.

Mirhedayatian, S. M., Azadi, M., \& Farzipoor Saen, R. (2014). A Novel Network Data Envelopment Analysis Model for Evaluating Green Supply Chain Management. International Journal of Production Economics, 147, 544-554.

Momeni, E., Tavana, M., Mirzagoltabar, H., \& Mirhedayatian, S. M. (2014). A New Fuzzy Network Slacks-Based DEA Model for Evaluating Performance of Supply Chains with Reverse Logistics. Journal of Intelligent \& Fuzzy Systems, 27(2), 793-804.

Nasseri, S. H., \& Khatir, M. A. (2019). Fuzzy Stochastic Undesirable Two-Stage Data Envelopment Analysis Models with Application to Banking Industry. Journal of Intelligent \& Fuzzy Systems, 37(5), 7047-7057.

Nosrat, A., Sanei, M., Payan, A., Hosseinzadeh Lotfi, F., \& Razavyan, S. (2019). Using Credibility Theory to Evaluate the Fuzzy Two-Stage DEA; Sensitivity and Stability Analysis. Journal of Intelligent \& Fuzzy Systems, 37(4), 5777-5796.

Olfat, L., \& Pishdar, M. (2017). Interval Type-2 Fuzzy Dynamic Network Data Envelopment Analysis with Undesirable Outputs Considering Double Frontiers: An Application to Iran Airports' Sustainability Evaluation. International Journal of Industrial Engineering, 24(6), 635-662.

Olfat, L., Amiri, M., Soufi, J. B., \& Pishdar, M. (2016). A Dynamic Network Efficiency Measurement of Airports Performance Considering Sustainable Development Concept: A Fuzzy Dynamic Network-DEA Approach. Journal of Air Transport Management, 57, 272-290.

Omrani, H., Keshavarz, M., \& Ghaderi, S. F. (2018). Evaluation of Supply Chain of a Shipping Company in Iran by a Fuzzy Relational Network Data Envelopment Analysis Model. Scientia Iranica, 25(2), 868-890.

Ostovan, S., Mozaffari, M. R., Jamshidi, A., \& Gerami, J. (2020). Evaluation of TwoStage Networks Based on Average Efficiency Using DEA and DEA-R with Fuzzy Data. International Journal of Fuzzy Systems, 22, 1665-1678.

Peykani, P., Mohammadi, E., Emrouznejad, A., Pishvaee, M. S., \& RostamyMalkhalifeh, M. (2019). Fuzzy Data Envelopment Analysis: An Adjustable Approach. Expert Systems with Applications, 136, 439-452. 
Peykani, P., Mohammadi, E., Farzipoor Saen, R., Sadjadi, S. J., \& RostamyMalkhalifeh, M. (2020). Data Envelopment Analysis and Robust Optimization: A Review. Expert Systems, e12534. https://doi.org/10.1111/exsy.12534

Peykani, P., Mohammadi, E., Pishvaee, M. S., Rostamy-Malkhalifeh, M., \& Jabbarzadeh, A. (2018). A Novel Fuzzy Data Envelopment Analysis Based on Robust Possibilistic Programming: Possibility, Necessity and Credibility-Based Approaches. RAIRO-Operations Research, 52(4), 1445-1463.

Premachandra, I. M., Zhu, J., Watson, J., \& Galagedera, D. U. (2012). Best-Performing US Mutual Fund Families from 1993 to 2008: Evidence from a Novel Two-Stage DEA Model for Efficiency Decomposition. Journal of Banking E Finance, 36(12), 3302-3317.

Rostamy-Malkhalifeh, M., \& Mollaeian, E. (2012). Evaluating Performance Supply Chain by a New Non-Radial Network DEA Model with Fuzzy Data. Data Envelopment Analysis and Decision Science, 1-9.

Sarah, J., \& Khalili-Damghani, K. (2019). Fuzzy Type-II De-Novo Programming for Resource Allocation and Target Setting in Network Data Envelopment Analysis: A Natural Gas Supply Chain. Expert Systems with Applications, 117, 312-329.

Shermeh, H. E., Najafi, S. E., \& Alavidoost, M. H. (2016). A Novel Fuzzy Network SBM Model for Data Envelopment Analysis: A Case Study in Iran Regional Power Companies. Energy, 112, 686-697.

Shi, X., Emrouznejad, A., Jin, M., \& Yang, F. (2020). A new parallel fuzzy data envelopment analysis model for parallel systems with two components based on Stackelberg game theory. Fuzzy Optimization and Decision Making, 19, 311-332.

Shiraz, R. K., Tavana, M., Fukuyama, H., \& Di Caprio, D. (2017). Fuzzy chanceconstrained geometric programming: the possibility, necessity and credibility approaches. Operational Research, 17(1), 67-97.

Simsek, B., \& Tüysüz, F. (2018). An Application of Network Data Envelopment Analysis with Fuzzy Data for the Performance Evaluation in Cargo Sector. Journal of Enterprise Information Management, 31(4), 492-509.

Singh, S., \& Arya, A. (2019). Development of Two-Stage Parallel-Series System with Fuzzy Data: Fuzzy DEA Approach. SSRN 3424550. 
Soltanzadeh, E., \& Omrani, H. (2018). Dynamic Network Data Envelopment Analysis Model with Fuzzy Inputs and Outputs: An Application for Iranian Airlines. Applied Soft Computing, 63, 268-288.

Tabasi, M., Navabakhsh, M., Hafezalkotob, A., \& Tavakkoli-Moghaddam, R. (2019). Performance Evaluation Using Network Data Envelopment Analysis Approach with Game Theory under Mixed Grey-Fuzzy Uncertainty in Iran Khodro Company. International Transaction Journal of Engineering, Management, \& Applied Sciences $\mathcal{E}$ Technologies, 10(13), 10A13L.

Tavana, M., \& Khalili-Damghani, K. (2014). A New Two-Stage Stackelberg Fuzzy Data Envelopment Analysis Model. Measurement, 53, 277-296.

Tavana, M., Khalili-Damghani, K., Arteaga, F. J. S., Mahmoudi, R., \& Hafezalkotob, A. (2018). Efficiency Decomposition and Measurement in Two-Stage Fuzzy DEA Models Using a Bargaining Game Approach. Computers \& Industrial Engineering, 118, 394-408.

Wang, L., \& Yao, C. (2020). Non-radial Fuzzy Network DEA Model Based on Directional Distance Function and Application in Supply Chain Efficiency Evaluation. Proceedings of the Sixth International Forum on Decision Sciences, 251-273, Springer, Singapore.

Wang, W. K., Lu, W. M., \& Liu, P. Y. (2014). A Fuzzy Multi-Objective Two-Stage DEA Model for Evaluating the Performance of US Bank Holding Companies. Expert Systems with Applications, 41(9), 4290-4297.

Xia, Q., Liang, L., \& Yang, F. (2014). Integrating Fuzzy Intermediate Factors in Supply Chain Efficiency Evaluation. Performance Measurement with Fuzzy Data Envelopment Analysis, 243-254, Springer.

Xu, J., \& Zhou, X. (2011). Fuzzy-Like Multiple Objective Decision Making. Berlin: Springer.

Yousefi, S., Soltani, R., Saen, R. F., \& Pishvaee, M. S. (2017). A robust fuzzy possibilistic programming for a new network GP-DEA model to evaluate sustainable supply chains. Journal of Cleaner Production, 166, 537-549.

Zadeh, L. A. (1978). Fuzzy Sets as a Basis for a Theory of Possibility. Fuzzy Sets and Systems, 1(1), 3-28. 
Zhou, X., Wang, Y., Chai, J., Wang, L., Wang, S., \& Lev, B. (2019a). Sustainable Supply Chain Evaluation: A Dynamic Double Frontier Network DEA Model with Interval Type-2 Fuzzy Data. Information Sciences, 504, 394-421.

Zhou, X., Xu, Z., Chai, J., Yao, L., Wang, S., \& Lev, B. (2019b). Efficiency Evaluation for Banking Systems under Uncertainty: A Multi-Period Three-Stage DEA Model. Omega, 85, 68-82.

Zhou, X., Xu, Z., Yao, L., Tu, Y., Lev, B., \& Pedrycz, W. (2018). A novel Data Envelopment Analysis model for evaluating industrial production and environmental management system. Journal of Cleaner Production, 170, 773-788.

\section{Appendix A. NDEA Approach - Stage 2 Is More Important}

If assumed that the efficiency of the second stage is more important for the DM, $\Psi_{p}^{\text {Stage2 }}$ will be calculated by solving Model (A1) while $\Psi_{p}^{\text {Overall* }}$ is optimized from Model (8):

$$
\Psi_{p}^{\text {Stage2 }}=\operatorname{Max} \frac{\sum_{r=1}^{R} y_{r p} \mu_{r}}{\sum_{d=1}^{D} z_{d p} \gamma_{d}+\sum_{h=1}^{H} g_{h p} \pi_{h}}
$$

S.t. $\quad \frac{\sum_{k=1}^{K} f_{k j} \omega_{k}+\sum_{d=1}^{D} z_{d j} \gamma_{d}}{\sum_{i=1}^{I} x_{i j} \tau_{i}} \leq 1, \quad \forall j$

$$
\begin{gathered}
\frac{\sum_{r=1}^{R} y_{r j} \mu_{r}}{\sum_{d=1}^{D} z_{d j} \gamma_{d}+\sum_{h=1}^{H} g_{h j} \pi_{h}} \leq 1, \quad \forall j \\
\frac{\sum_{k=1}^{K} f_{k p} \omega_{k}+\sum_{d=1}^{D} z_{d p} \gamma_{d}+\sum_{r=1}^{R} y_{r p} \mu_{r}}{\sum_{i=1}^{I} x_{i p} \tau_{i}+\sum_{d=1}^{D} z_{d p} \gamma_{d}+\sum_{h=1}^{H} g_{h p} \pi_{h}}=\Psi_{p}^{\text {Overall* }^{*}} \\
\tau_{i}, \omega_{k}, \gamma_{d}, \pi_{h}, \mu_{r} \geq 0, \quad \forall i, k, d, h, r
\end{gathered}
$$


Like the previous fractional models in this study, by utilizing the Charnes and Cooper (1962) transformation, Model (A1) will be equivalent to Model (A2):

$$
\begin{aligned}
\Psi_{p}^{\text {Stage2 }}= & \operatorname{Max} \sum_{r=1}^{R} y_{r p} \mu_{r} \\
\text { S.t. } \quad & \sum_{d=1}^{D} z_{d p} \gamma_{d}+\sum_{h=1}^{H} g_{h p} \pi_{h}=1 \\
& \sum_{k=1}^{K} f_{k j} \omega_{k}+\sum_{d=1}^{D} z_{d j} \gamma_{d}-\sum_{i=1}^{I} x_{i j} \tau_{i} \leq 0, \quad \forall j \\
& \sum_{r=1}^{R} y_{r j} \mu_{r}-\sum_{d=1}^{D} z_{d j} \gamma_{d}-\sum_{h=1}^{H} g_{h j} \pi_{h} \leq 0, \quad \forall j \\
& \sum_{k=1}^{K} f_{k p} \omega_{k}+\sum_{d=1}^{D} z_{d p} \gamma_{d}+\sum_{r=1}^{R} y_{r p} \mu_{r}-\Psi_{p}^{\text {Overall* }} \sum_{i=1}^{I} x_{i p} \tau_{i}=\Psi_{p}^{\text {Overall* }} \\
& \tau_{i}, \omega_{k}, \gamma_{d}, \pi_{h}, \mu_{r} \geq 0, \quad \forall i, k, d, h, r
\end{aligned}
$$

Finally, after calculating $\Psi_{p}^{\text {Stage2* }}$ using the Model (A2), the efficiency score of the stage 1 is obtained from Equation (A3):

$$
\Psi_{p}^{\text {Stage }^{*}}=\frac{\Psi_{p}^{\text {Overall }^{*}}-\xi_{2}^{*} \Psi_{p}^{\text {Stage2* }}}{\xi_{1}^{*}}
$$

It should be mentioned that $\xi_{1}^{*}$ and $\xi_{2}^{*}$ are optimal weights that obtained from Model (8) using Equations (5) and (6).

\section{Appendix B. Integrated FNDEA Models}

Please note that using a binary variable $(\dagger)$ and a sufficient big number $(\mathrm{M})$, the linearization of incompatible constraints for the $\alpha$ greater or less than $\beta$ are made. Accordingly, by integrating Models (20) and (21), a new version of proposed AFNDEA approach to measure of overall efficiency score $\Psi_{p(\alpha, \beta)}^{\text {Overall }}$ under fuzzy data is introduced as Model (B1): 
S.t. $\quad \sum_{k=1}^{K}\left(\left(\frac{\alpha}{\beta}\right) f_{k p}^{3}+\left(\frac{\beta-\alpha}{\beta}\right) f_{k p}^{4}\right) \omega_{k}+\sum_{d=1}^{D}\left(\left(\frac{\alpha}{\beta}\right) z_{d p}^{3}+\left(\frac{\beta-\alpha}{\beta}\right) z_{d p}^{4}\right) \gamma_{d}$

$+\sum_{r=1}^{R}\left(\left(\frac{\alpha}{\beta}\right) y_{r p}^{3}+\left(\frac{\beta-\alpha}{\beta}\right) y_{r p}^{4}\right) \mu_{r} \geq \Omega-\dagger \mathrm{M}$

$\sum_{k=1}^{K}\left(\left(\frac{\alpha-\beta}{1-\beta}\right) f_{k p}^{1}+\left(\frac{1-\alpha}{1-\beta}\right) f_{k p}^{2}\right) \omega_{k}+\sum_{d=1}^{D}\left(\left(\frac{\alpha-\beta}{1-\beta}\right) z_{d p}^{1}+\left(\frac{1-\alpha}{1-\beta}\right) z_{d p}^{2}\right) \gamma_{d}$

$+\sum_{r=1}^{R}\left(\left(\frac{\alpha-\beta}{1-\beta}\right) y_{r p}^{1}+\left(\frac{1-\alpha}{1-\beta}\right) y_{r p}^{2}\right) \mu_{r} \geq \Omega-(1-\dagger) \mathrm{M}$

$\sum_{i=1}^{I}\left(\left(\frac{\beta-\alpha}{\beta}\right) x_{i p}^{1}+\left(\frac{\alpha}{\beta}\right) x_{i p}^{2}\right) \tau_{i}+\sum_{d=1}^{D}\left(\left(\frac{\beta-\alpha}{\beta}\right) z_{d p}^{1}+\left(\frac{\alpha}{\beta}\right) z_{d p}^{2}\right) \gamma_{d}$

$+\sum_{h=1}^{H}\left(\left(\frac{\beta-\alpha}{\beta}\right) g_{h p}^{1}+\left(\frac{\alpha}{\beta}\right) g_{h p}^{2}\right) \pi_{h} \leq 1+\dagger \mathrm{M}$

$\sum_{i=1}^{I}\left(\left(\frac{1-\alpha}{1-\beta}\right) x_{i p}^{3}+\left(\frac{\alpha-\beta}{1-\beta}\right) x_{i p}^{4}\right) \tau_{i}+\sum_{d=1}^{D}\left(\left(\frac{1-\alpha}{1-\beta}\right) z_{d p}^{3}+\left(\frac{\alpha-\beta}{1-\beta}\right) z_{d p}^{4}\right) \gamma_{d}$

$+\sum_{h=1}^{H}\left(\left(\frac{1-\alpha}{1-\beta}\right) g_{h p}^{3}+\left(\frac{\alpha-\beta}{1-\beta}\right) g_{h p}^{4}\right) \pi_{h} \leq 1+(1-\dagger) \mathrm{M}$

$\sum_{k=1}^{K}\left(\left(\frac{\beta-\alpha}{\beta}\right) f_{k j}^{1}+\left(\frac{\alpha}{\beta}\right) f_{k j}^{2}\right) \omega_{k}+\sum_{d=1}^{D}\left(\left(\frac{\beta-\alpha}{\beta}\right) z_{d j}^{1}+\left(\frac{\alpha}{\beta}\right) z_{d j}^{2}\right) \gamma_{d}$

$-\sum_{i=1}^{I}\left(\left(\frac{\alpha}{\beta}\right) x_{i j}^{3}+\left(\frac{\beta-\alpha}{\beta}\right) x_{i j}^{4}\right) \tau_{i} \leq \uparrow \mathrm{M}, \quad \forall j$

$\sum_{k=1}^{K}\left(\left(\frac{1-\alpha}{1-\beta}\right) f_{k j}^{3}+\left(\frac{\alpha-\beta}{1-\beta}\right) f_{k j}^{4}\right) \omega_{k}+\sum_{d=1}^{D}\left(\left(\frac{1-\alpha}{1-\beta}\right) z_{d j}^{3}+\left(\frac{\alpha-\beta}{1-\beta}\right) z_{d j}^{4}\right) \gamma_{d}$

$-\sum_{i=1}^{I}\left(\left(\frac{\alpha-\beta}{1-\beta}\right) x_{i j}^{1}+\left(\frac{1-\alpha}{1-\beta}\right) x_{i j}^{2}\right) \tau_{i} \leq(1-\dagger) \mathrm{M}, \quad \forall j$

$\sum_{r=1}^{R}\left(\left(\frac{\beta-\alpha}{\beta}\right) y_{r j}^{1}+\left(\frac{\alpha}{\beta}\right) y_{r j}^{2}\right) \mu_{r}-\sum_{d=1}^{D}\left(\left(\frac{\alpha}{\beta}\right) z_{d j}^{3}+\left(\frac{\beta-\alpha}{\beta}\right) z_{d j}^{4}\right) \gamma_{d}$

$-\sum_{h=1}^{H}\left(\left(\frac{\alpha}{\beta}\right) g_{h j}^{3}+\left(\frac{\beta-\alpha}{\beta}\right) g_{h j}^{4}\right) \pi_{h} \leq \uparrow \mathrm{M}, \quad \forall j$

$\sum_{r=1}^{R}\left(\left(\frac{1-\alpha}{1-\beta}\right) y_{r j}^{3}+\left(\frac{\alpha-\beta}{1-\beta}\right) y_{r j}^{4}\right) \mu_{r}-\sum_{d=1}^{D}\left(\left(\frac{\alpha-\beta}{1-\beta}\right) z_{d j}^{1}+\left(\frac{1-\alpha}{1-\beta}\right) z_{d j}^{2}\right) \gamma_{d}$

$-\sum_{h=1}^{H}\left(\left(\frac{\alpha-\beta}{1-\beta}\right) g_{h j}^{1}+\left(\frac{1-\alpha}{1-\beta}\right) g_{h j}^{2}\right) \pi_{h} \leq(1-\dagger) \mathrm{M}, \quad \forall j$

$\alpha \leq \beta+\uparrow \mathrm{M}$

$\alpha>\beta-(1-\dagger) \mathrm{M} \quad \alpha \leq \beta+\dagger \mathrm{M}$ 
$\dagger \in\{0,1\}$

$\tau_{i}, \omega_{k}, \gamma_{d}, \pi_{h}, \mu_{r} \geq 0, \quad \forall i, k, d, h, r$

Also, the efficiency of the first stage $\Psi_{p(\alpha, \beta)}^{\text {Stage } 1}$ in the presence of fuzzy data can be estimated by solving Model (B2) while $\Psi_{p(\alpha, \beta)}^{\text {Overal** }}$ is obtained from Model (B1):

$\Psi_{p(\alpha, \beta)}^{\text {Stagel }}=\operatorname{Max} \Omega$

S.t. $\quad \sum_{k=1}^{K}\left(\left(\frac{\alpha}{\beta}\right) f_{k p}^{3}+\left(\frac{\beta-\alpha}{\beta}\right) f_{k p}^{4}\right) \omega_{k}$

$+\sum_{d=1}^{D}\left(\left(\frac{\alpha}{\beta}\right) z_{d p}^{3}+\left(\frac{\beta-\alpha}{\beta}\right) z_{d p}^{4}\right) \gamma_{d} \geq \Omega-\dagger \mathrm{M}$

$\sum_{k=1}^{K}\left(\left(\frac{\alpha-\beta}{1-\beta}\right) f_{k p}^{1}+\left(\frac{1-\alpha}{1-\beta}\right) f_{k p}^{2}\right) \omega_{k}$

$+\sum_{d=1}^{D}\left(\left(\frac{\alpha-\beta}{1-\beta}\right) z_{d p}^{1}+\left(\frac{1-\alpha}{1-\beta}\right) z_{d p}^{2}\right) \gamma_{d} \geq \Omega-(1-\dagger) \mathrm{M}$

$\sum_{i=1}^{I}\left(\left(\frac{\beta-\alpha}{\beta}\right) x_{i p}^{1}+\left(\frac{\alpha}{\beta}\right) x_{i p}^{2}\right) \tau_{i} \leq 1+\dagger \mathrm{M}$

$\sum_{i=1}^{I}\left(\left(\frac{1-\alpha}{1-\beta}\right) x_{i p}^{3}+\left(\frac{\alpha-\beta}{1-\beta}\right) x_{i p}^{4}\right) \tau_{i} \leq 1+(1-\dagger) \mathrm{M}$

$\sum_{k=1}^{K}\left(\left(\frac{\beta-\alpha}{\beta}\right) f_{k j}^{1}+\left(\frac{\alpha}{\beta}\right) f_{k j}^{2}\right) \omega_{k}+\sum_{d=1}^{D}\left(\left(\frac{\beta-\alpha}{\beta}\right) z_{d j}^{1}+\left(\frac{\alpha}{\beta}\right) z_{d j}^{2}\right) \gamma_{d}$

$-\sum_{i=1}^{I}\left(\left(\frac{\alpha}{\beta}\right) x_{i j}^{3}+\left(\frac{\beta-\alpha}{\beta}\right) x_{i j}^{4}\right) \tau_{i} \leq \dagger \mathrm{M}, \quad \forall j$

$\sum_{k=1}^{K}\left(\left(\frac{1-\alpha}{1-\beta}\right) f_{k j}^{3}+\left(\frac{\alpha-\beta}{1-\beta}\right) f_{k j}^{4}\right) \omega_{k}+\sum_{d=1}^{D}\left(\left(\frac{1-\alpha}{1-\beta}\right) z_{d j}^{3}+\left(\frac{\alpha-\beta}{1-\beta}\right) z_{d j}^{4}\right) \gamma_{d}$

$-\sum_{i=1}^{I}\left(\left(\frac{\alpha-\beta}{1-\beta}\right) x_{i j}^{1}+\left(\frac{1-\alpha}{1-\beta}\right) x_{i j}^{2}\right) \tau_{i} \leq(1-\dagger) \mathrm{M}, \quad \forall j$

$\sum_{r=1}^{R}\left(\left(\frac{\beta-\alpha}{\beta}\right) y_{r j}^{1}+\left(\frac{\alpha}{\beta}\right) y_{r j}^{2}\right) \mu_{r}-\sum_{d=1}^{D}\left(\left(\frac{\alpha}{\beta}\right) z_{d j}^{3}+\left(\frac{\beta-\alpha}{\beta}\right) z_{d j}^{4}\right) \gamma_{d}$

$-\sum_{h=1}^{H}\left(\left(\frac{\alpha}{\beta}\right) g_{h j}^{3}+\left(\frac{\beta-\alpha}{\beta}\right) g_{h j}^{4}\right) \pi_{h} \leq \uparrow \mathrm{M}, \quad \forall j$ 


$$
\begin{aligned}
& \sum_{r=1}^{R}\left(\left(\frac{1-\alpha}{1-\beta}\right) y_{r j}^{3}+\left(\frac{\alpha-\beta}{1-\beta}\right) y_{r j}^{4}\right) \mu_{r}-\sum_{d=1}^{D}\left(\left(\frac{\alpha-\beta}{1-\beta}\right) z_{d j}^{1}+\left(\frac{1-\alpha}{1-\beta}\right) z_{d j}^{2}\right) \gamma_{d} \\
& -\sum_{h=1}^{H}\left(\left(\frac{\alpha-\beta}{1-\beta}\right) g_{h j}^{1}+\left(\frac{1-\alpha}{1-\beta}\right) g_{h j}^{2}\right) \pi_{h} \leq(1-\dagger) \mathrm{M}, \quad \forall j \\
& \sum_{k=1}^{K}\left(\left(\frac{\alpha}{\beta}\right) f_{k p}^{3}+\left(\frac{\beta-\alpha}{\beta}\right) f_{k p}^{4}\right) \omega_{k}+\sum_{d=1}^{D}\left(\left(\frac{\alpha}{\beta}\right) z_{d p}^{3}+\left(\frac{\beta-\alpha}{\beta}\right) z_{d p}^{4}\right) \gamma_{d} \\
& +\sum_{r=1}^{R}\left(\left(\frac{\alpha}{\beta}\right) y_{r p}^{3}+\left(\frac{\beta-\alpha}{\beta}\right) y_{r p}^{4}\right) \mu_{r}-\Psi_{p(\alpha, \beta)}^{\text {Overal* }} \sum_{d=1}^{D}\left(\left(\frac{\beta-\alpha}{\beta}\right) z_{d p}^{1}+\left(\frac{\alpha}{\beta}\right) z_{d p}^{2}\right) \gamma_{d} \\
& -\Psi_{p(\alpha, \beta)}^{\text {Overal } *} \sum_{h=1}^{H}\left(\left(\frac{\beta-\alpha}{\beta}\right) g_{h p}^{1}+\left(\frac{\alpha}{\beta}\right) g_{h p}^{2}\right) \pi_{h} \geq \Psi_{p(\alpha, \beta)}^{\text {Overall* }}-\dagger \mathrm{M} \\
& \sum_{k=1}^{K}\left(\left(\frac{\alpha-\beta}{1-\beta}\right) f_{k p}^{1}+\left(\frac{1-\alpha}{1-\beta}\right) f_{k p}^{2}\right) \omega_{k}+\sum_{d=1}^{D}\left(\left(\frac{\alpha-\beta}{1-\beta}\right) z_{d p}^{1}+\left(\frac{1-\alpha}{1-\beta}\right) z_{d p}^{2}\right) \gamma_{d} \\
& +\sum_{r=1}^{R}\left(\left(\frac{\alpha-\beta}{1-\beta}\right) y_{r p}^{1}+\left(\frac{1-\alpha}{1-\beta}\right) y_{r p}^{2}\right) \mu_{r}-\Psi_{p(\alpha, \beta)}^{\text {Overal } *} \sum_{d=1}^{D}\left(\left(\frac{1-\alpha}{1-\beta}\right) z_{d p}^{3}+\left(\frac{\alpha-\beta}{1-\beta}\right) z_{d p}^{4}\right) \gamma_{d} \\
& -\Psi_{p(\alpha, \beta)}^{\text {Overall* }} \sum_{h=1}^{H}\left(\left(\frac{1-\alpha}{1-\beta}\right) g_{h p}^{3}+\left(\frac{\alpha-\beta}{1-\beta}\right) g_{h p}^{4}\right) \pi_{h} \geq \Psi_{p(\alpha, \beta)}^{\text {Overal* }}-(1-\dagger) \mathrm{M} \\
& \alpha \leq \beta+\uparrow \mathrm{M} \\
& \alpha>\beta-(1-\dagger) \mathrm{M} \\
& \dagger \in\{0,1\} \\
& \tau_{i}, \omega_{k}, \gamma_{d}, \pi_{h}, \mu_{r} \geq 0, \quad \forall i, k, d, h, r
\end{aligned}
$$

It should be mentioned that Model (B2) is final result of the integration of Models (22) and (23).

\section{Appendix C. FNDEA Approach - Stage 2 Is More Important}

To consider the uncertainty on all data, Model (A2) for fuzzy observations can be written as Model (C1):

$$
\begin{array}{ll}
\Psi_{p}^{\text {Stage2 }}= & \text { Max } \Omega \\
\text { S.t. } & \sum_{r=1}^{R} \tilde{y}_{r p} \mu_{r} \geq \Omega \\
& \sum_{d=1}^{D} \tilde{z}_{d p} \gamma_{d}+\sum_{h=1}^{H} \tilde{g}_{h p} \pi_{h} \leq 1
\end{array}
$$




$$
\begin{aligned}
& \sum_{k=1}^{K} \tilde{f}_{k j} \omega_{k}+\sum_{d=1}^{D} \tilde{z}_{d j} \gamma_{d}-\sum_{i=1}^{I} \tilde{x}_{i j} \tau_{i} \leq 0, \quad \forall j \\
& \sum_{r=1}^{R} \tilde{y}_{r j} \mu_{r}-\sum_{d=1}^{D} \tilde{z}_{d j} \gamma_{d}-\sum_{h=1}^{H} \tilde{g}_{h j} \pi_{h} \leq 0, \quad \forall j \\
& \sum_{k=1}^{K} \tilde{f}_{k p} \omega_{k}+\sum_{d=1}^{D} \tilde{z}_{d p} \gamma_{d}+\sum_{r=1}^{R} \tilde{y}_{r p} \mu_{r}-\Psi_{p}^{\text {Overall }} \sum_{i=1}^{I} \tilde{x}_{i p} \tau_{i} \geq \Psi_{p}^{\text {Overall* }} \\
& \tau_{i}, \omega_{k}, \gamma_{d}, \pi_{h}, \mu_{r} \geq 0, \quad \forall i, k, d, h, r
\end{aligned}
$$

The efficiency of the second stage under fuzzy data is calculated by solving Model (C2) while $\Psi_{p(\alpha, \beta)}^{\text {Overal* }}$ is obtained from Models (20) and (21):

S.t. $\quad \sum_{r=1}^{R}\left(\left(\frac{\alpha}{\beta}\right) y_{r p}^{3}+\left(\frac{\beta-\alpha}{\beta}\right) y_{r p}^{4}\right) \mu_{r} \geq \Omega-\dagger \mathrm{M}$

$$
\begin{aligned}
& \sum_{r=1}^{R}\left(\left(\frac{\alpha-\beta}{1-\beta}\right) y_{r p}^{1}+\left(\frac{1-\alpha}{1-\beta}\right) y_{r p}^{2}\right) \mu_{r} \geq \Omega-(1-\dagger) \mathrm{M} \\
& \sum_{d=1}^{D}\left(\left(\frac{\beta-\alpha}{\beta}\right) z_{d p}^{1}+\left(\frac{\alpha}{\beta}\right) z_{d p}^{2}\right) \gamma_{d} \\
+ & \sum_{h=1}^{H}\left(\left(\frac{\beta-\alpha}{\beta}\right) g_{h p}^{1}+\left(\frac{\alpha}{\beta}\right) g_{h p}^{2}\right) \pi_{h} \leq 1+\dagger \mathrm{M} \\
& \sum_{d=1}^{D}\left(\left(\frac{1-\alpha}{1-\beta}\right) z_{d p}^{3}+\left(\frac{\alpha-\beta}{1-\beta}\right) z_{d p}^{4}\right) \gamma_{d} \\
+ & \sum_{h=1}^{H}\left(\left(\frac{1-\alpha}{1-\beta}\right) g_{h p}^{3}+\left(\frac{\alpha-\beta}{1-\beta}\right) g_{h p}^{4}\right) \pi_{h} \leq 1+(1-\dagger) \mathrm{M} \\
& \sum_{k=1}^{K}\left(\left(\frac{\beta-\alpha}{\beta}\right) f_{k j}^{1}+\left(\frac{\alpha}{\beta}\right) f_{k j}^{2}\right) \omega_{k}+\sum_{d=1}^{D}\left(\left(\frac{\beta-\alpha}{\beta}\right) z_{d j}^{1}+\left(\frac{\alpha}{\beta}\right) z_{d j}^{2}\right) \gamma_{d} \\
& -\sum_{i=1}^{I}\left(\left(\frac{\alpha}{\beta}\right) x_{i j}^{3}+\left(\frac{\beta-\alpha}{\beta}\right) x_{i j}^{4}\right) \tau_{i} \leq \dagger \mathrm{M}, \quad \forall j \\
& \sum_{k=1}^{K}\left(\left(\frac{1-\alpha}{1-\beta}\right) f_{k j}^{3}+\left(\frac{\alpha-\beta}{1-\beta}\right) f_{k j}^{4}\right) \omega_{k}+\sum_{d=1}^{D}\left(\left(\frac{1-\alpha}{1-\beta}\right) z_{d j}^{3}+\left(\frac{\alpha-\beta}{1-\beta}\right) z_{d j}^{4}\right) \gamma_{d} \\
- & \sum_{i=1}^{I}\left(\left(\frac{\alpha-\beta}{1-\beta}\right) x_{i j}^{1}+\left(\frac{1-\alpha}{1-\beta}\right) x_{i j}^{2}\right) \tau_{i} \leq(1-\dagger) \mathrm{M}, \forall j \\
& (1-\beta
\end{aligned}
$$




$$
\begin{aligned}
& \sum_{r=1}^{R}\left(\left(\frac{\beta-\alpha}{\beta}\right) y_{r j}^{1}+\left(\frac{\alpha}{\beta}\right) y_{r j}^{2}\right) \mu_{r}-\sum_{d=1}^{D}\left(\left(\frac{\alpha}{\beta}\right) z_{d j}^{3}+\left(\frac{\beta-\alpha}{\beta}\right) z_{d j}^{4}\right) \gamma_{d} \\
& -\sum_{h=1}^{H}\left(\left(\frac{\alpha}{\beta}\right) g_{h j}^{3}+\left(\frac{\beta-\alpha}{\beta}\right) g_{h j}^{4}\right) \pi_{h} \leq \uparrow \mathrm{M}, \quad \forall j \\
& \sum_{r=1}^{R}\left(\left(\frac{1-\alpha}{1-\beta}\right) y_{r j}^{3}+\left(\frac{\alpha-\beta}{1-\beta}\right) y_{r j}^{4}\right) \mu_{r}-\sum_{d=1}^{D}\left(\left(\frac{\alpha-\beta}{1-\beta}\right) z_{d j}^{1}+\left(\frac{1-\alpha}{1-\beta}\right) z_{d j}^{2}\right) \gamma_{d} \\
& -\sum_{h=1}^{H}\left(\left(\frac{\alpha-\beta}{1-\beta}\right) g_{h j}^{1}+\left(\frac{1-\alpha}{1-\beta}\right) g_{h j}^{2}\right) \pi_{h} \leq(1-\dagger) \mathrm{M}, \quad \forall j \\
& \sum_{k=1}^{K}\left(\left(\frac{\alpha}{\beta}\right) f_{k p}^{3}+\left(\frac{\beta-\alpha}{\beta}\right) f_{k p}^{4}\right) \omega_{k} \\
& +\sum_{d=1}^{D}\left(\left(\frac{\alpha}{\beta}\right) z_{d p}^{3}+\left(\frac{\beta-\alpha}{\beta}\right) z_{d p}^{4}\right) \gamma_{d}+\sum_{r=1}^{R}\left(\left(\frac{\alpha}{\beta}\right) y_{r p}^{3}+\left(\frac{\beta-\alpha}{\beta}\right) y_{r p}^{4}\right) \mu_{r} \\
& -\Psi_{p(\alpha, \beta)}^{\text {Overall* }} \sum_{i=1}^{I}\left(\left(\frac{\beta-\alpha}{\beta}\right) x_{i p}^{1}+\left(\frac{\alpha}{\beta}\right) x_{i p}^{2}\right) \tau_{i} \geq \Psi_{p(\alpha, \beta)}^{\text {Overall* }}-\dagger \mathrm{M} \\
& \sum_{k=1}^{K}\left(\left(\frac{\alpha-\beta}{1-\beta}\right) f_{k p}^{1}+\left(\frac{1-\alpha}{1-\beta}\right) f_{k p}^{2}\right) \omega_{k} \\
& +\sum_{d=1}^{D}\left(\left(\frac{\alpha-\beta}{1-\beta}\right) z_{d p}^{1}+\left(\frac{1-\alpha}{1-\beta}\right) z_{d p}^{2}\right) \gamma_{d}+\sum_{r=1}^{R}\left(\left(\frac{\alpha-\beta}{1-\beta}\right) y_{r p}^{1}+\left(\frac{1-\alpha}{1-\beta}\right) y_{r p}^{2}\right) \mu_{r} \\
& -\Psi_{p(\alpha, \beta)}^{\text {Overall* }} \sum_{i=1}^{I}\left(\left(\frac{1-\alpha}{1-\beta}\right) x_{i p}^{3}+\left(\frac{\alpha-\beta}{1-\beta}\right) x_{i p}^{4}\right) \tau_{i} \geq \Psi_{p(\alpha, \beta)}^{\text {Overall* }}-(1-\dagger) \mathrm{M} \\
& \alpha \leq \beta+\dagger \mathrm{M} \\
& \alpha>\beta-(1-\dagger) \mathrm{M} \\
& \dagger \in\{0,1\} \\
& \tau_{i}, \omega_{k}, \gamma_{d}, \pi_{h}, \mu_{r} \geq 0, \quad \forall i, k, d, h, r
\end{aligned}
$$

Finally, after calculating $\Psi_{p(\alpha, \beta)}^{\text {Stage* }}$ from Model (C2), the efficiency score of stage 1 is measured using Equation (C3):

$$
\Psi_{p(\alpha, \beta)}^{\text {Stage }^{*}}=\frac{\Psi_{p(\alpha, \beta)}^{\text {Overall* }}-\xi_{2(\alpha, \beta)}^{*} \Psi_{p(\alpha, \beta)}^{\text {Stage } 2^{*}}}{\xi_{1(\alpha, \beta)}^{*}}
$$

Accordingly, Model (C2) and Equation (C3) can be applied instead of Models (22) and (23) as well as Equation (24), for situation that stage 2 is more important than stage 1. 